\title{
Superconductivity on the surface of topological insulators and in two-dimensional noncentrosymmetric materials
}

\author{
Luiz Santos, ${ }^{1}$ Titus Neupert, ${ }^{2}$ Claudio Chamon, ${ }^{3}$ and Christopher Mudry ${ }^{4}$ \\ ${ }^{1}$ Department of Physics, Harvard University, 17 Oxford Street, Cambridge, Massachusetts 02138, USA \\ ${ }^{2}$ Condensed Matter Theory Laboratory, RIKEN, Wako, Saitama 351-0198, Japan \\ ${ }^{3}$ Physics Department, Boston University, Boston, Massachusetts 02215, USA \\ ${ }^{4}$ Condensed Matter Theory Group, Paul Scherrer Institute, CH-5232 Villigen PSI, Switzerland \\ (Received 16 November 2009; revised manuscript received 18 March 2010; published 4 May 2010)
}

\begin{abstract}
We study the superconducting instabilities of a single species of two-dimensional Rashba-Dirac fermions, as it pertains to the surface of a three-dimensional time-reversal symmetric topological band insulator. We also discuss the similarities as well as the differences between this problem and that of superconductivity in two-dimensional time-reversal symmetric noncentrosymmetric materials with spin-orbit interactions. The superconducting order parameter has both $s$-wave and $p$-wave components, even when the superconducting pair potential only transfers either pure singlet or pure triplet pairs of electrons in and out of the condensate, a corollary to the nonconservation of spin due to the spin-orbit coupling. We identify one single superconducting regime in the case of superconductivity in the topological surface states (Rashba-Dirac limit), irrespective of the relative strength between singlet and triplet pair potentials. In contrast, in the Fermi limit relevant to the noncentrosymmetric materials we find two regimes depending on the value of the chemical potential and the relative strength between singlet and triplet potentials. We construct explicitly the Majorana bound states in these regimes. In the single regime for the case of the Rashba-Dirac limit, there exists one and only one Majorana fermion bound to the core of an isolated vortex. In the Fermi limit, there are always an even number ( 0 or 2 depending on the regime) of Majorana fermions bound to the core of an isolated vortex. In all cases, the vorticity required to bind Majorana fermions is quantized in units of the flux quantum, in contrast to the half flux in the case of two-dimensional $p_{x} \pm i p_{y}$ superconductors that break time-reversal symmetry.
\end{abstract}

DOI: 10.1103/PhysRevB.81.184502

PACS number(s): 74.90.+n, 74.20.Rp, 74.25.Ha

\section{INTRODUCTION}

$\mathrm{Bi}_{2} \mathrm{Se}_{3}$ is an inversion-symmetric layered band insulator with a bulk gap estimated to be $0.35 \mathrm{eV}^{1-3}$ Densityfunctional theory predicts that $\mathrm{Bi}_{2} \mathrm{Se}_{3}$ supports a single Rashba-Dirac cone of gapless surface states, a prediction that has been verified using angle-resolved photoemission spectroscopy. ${ }^{3,4}$ This remarkable attribute of $\mathrm{Bi}_{2} \mathrm{Se}_{3}$, which has otherwise only been observed in the insulating alloys $\mathrm{Bi}_{1-x} \mathrm{Sb}_{x}$ so far, ${ }^{5,6}$ is the defining property of a threedimensional (3D) time-reversal symmetric (TRS) topological band insulator. ${ }^{7-9}$ In a recent work, Hor et al. ${ }^{10}$ have reported the observation of strongly type II superconductivity in $\mathrm{Cu}_{x} \mathrm{Bi}_{2} \mathrm{Se}_{3}$ below $3.8 \mathrm{~K}$ when $\mathrm{Cu}$ is intercalated between the $\mathrm{Bi}_{2} \mathrm{Se}_{3}$ layers. They have also proposed to use $\mathrm{Cu}_{x} \mathrm{Bi}_{2} \mathrm{Se}_{3}$ as a mean to induce superconducting correlations for the TRS topological surface states by the proximity effect.

The surface states in a 3D TRS topological band insulator are reminiscent of the Bloch states of graphene in that, in both cases, their density of states vanishes linearly at the so-called Rashba-Dirac point. ${ }^{11}$ However, they differ in a fundamental way from those in graphene. For example, the surface of $\mathrm{Bi}_{2} \mathrm{Se}_{3}$ supports one Rashba-Dirac cone as opposed to two in graphene. This difference is a manifestation of the fact that inversion symmetry is maximally broken on the surface of $\mathrm{Bi}_{2} \mathrm{Se}_{3}$ in that the kinetic energy is dominantly of the Rashba type, whereas the spin-orbit coupling is for all intent and purposes negligible for graphene. Consequently, the surface states of a 3D TRS topological band insulator are not localized by weak TRS disorder, ${ }^{12-15}$ whereas Anderson localization rules in graphene. ${ }^{16}$
Another difference with graphene, as we shall show in this paper as a warm up, is that all states in the Rashba-Dirac sea contribute to the Pauli magnetic susceptibility, which is anisotropic in that the in-plane and out-of-plane components differ by a factor of 2 . For comparison, the Pauli magnetic susceptibility is isotropic in spin space and proportional to the density of states at the Fermi surface in any electron gas (including graphene) with small breaking of the spin-rotation symmetry (SRS). This anisotropy and the fact that the Pauli susceptibility does not only depend on the density of states at the Fermi level could potentially be used as a simple diagnostic of a limit in which the Rashba coupling is the largest energy scale.

The main emphasis of this paper will be on the superconducting instabilities of the surface states in a 3D TRS topological band insulator and on those in close relatives, i.e., two-dimensional (2D) TRS noncentrosymmetric superconductors in a regime that has been little studied so far. The theoretical studies of noncentrosymmetric superconductors with TRS usually assume the hierarchy of energy scales

$$
t \gg \alpha \gg \Delta,
$$

where $t$ is the inversion-symmetric band width, $\alpha>0$ is the spin-orbit coupling that preserves TRS but breaks SRS, and $\Delta>0$ is the single-particle superconducting gap. ${ }^{17-25}$ The regime

$$
\alpha \gg \Delta \gg t
$$

is the one that applies to intrinsic superconducting instabilities of the surface states in a 3D TRS topological band insu- 
lator. We will address the question of whether interesting phenomena associated to superconductivity occur upon exchanging the hierarchies (1.1) and (1.2).

In the same way that (2D) TRS band insulators have been classified according to their topological character, ${ }^{26-30}$ Bogoliubov-de-Gennes (BdG) superconductors have also been given topological attributes whenever they support gapless boundary states in confined geometries. ${ }^{31-33}$ A necessary (but not sufficient) condition for a 2D TRS superconductor to be topologically nontrivial is that it is noncentrosymmetric, or, equivalently, that it breaks SRS. According to Refs. 31 and 32, a sufficient condition is that, for any weak and local TRS static disorder, a 2D TRS superconductor in an infinitely long strip geometry supports an odd number of Kramers' doublets of gapless edge states of which at least one wave function is extended along the edge (see also Refs. 34-36 for varying alternative criteria).

Applying this definition of a topological superconducting phase to the superconducting instabilities of surface states in a 3D TRS topological band insulator immediately leads to a paradox: What is the meaning of the boundary of a boundary? A more meaningful question to ask might be: What are the spectral properties of TRS-breaking vortices if the surface states in a 3D TRS topological band insulator support a type II superconducting order? Do they bind mid gap states generically, zero modes in particular, or not? These are questions that we address in this paper.

Defects in a type II superconductor are vortices. On the one hand, Caroli et al. ${ }^{37}$ have shown that vortices in an $s$-wave TRS and SRS superconductor bind nonvanishingenergy bound states with a mean level spacing of the order of the superconducting gap squared divided by the Fermi energy. On the other hand, Jackiw and Rossi in Ref. 38 found a single bound state that is exponentially localized around the core of a unit-flux vortex in a 2D s-wave relativistic superconductor with a vanishing density of states (Rashba-Dirac point). The energy of this bound state is precisely pinned to the Fermi energy (see also Ref. 39 for the corresponding index theorem and Refs. 40 and 41 for examples of nonrelativistic zero modes bound to vortices). A midgap state bound to the core of a vortex does not carry an electric charge, for it is an eigenstate of the generator of the particlehole symmetry (PHS) obeyed by any BdG Hamiltonian. It is thus charge neutral and as such is a physical realization of a Majorana fermion. Majorana fermions were also found to be exponentially localized to the core of a vortex in a $p_{x} \pm i p_{y}$ type II superconductor by Read and Green and by Ivanov in Refs. 42 and 43, respectively. More importantly, they showed that these Majorana fermions obey non-Abelian braiding statistics. Theoretical proposals to nucleate Majorana fermions have been made relying on 2D TRS noncentrosymmetric superconductors, ${ }^{44-46}$ or on proximity effects at the $2 \mathrm{D}$ interface between band insulators, superconductors, and ferromagnets. ${ }^{47-49}$

We will show in this paper that, when the dispersion is Rashba-Dirac like, there is a single zero mode bound to the core of an isolated vortex with unit circulation, and thus a single Majorana bound state. The mechanism, in the case of singlet pairing, is precisely that of Jackiw and Rossi. ${ }^{38}$ This zero mode remains for arbitrary ratios of triplet and singlet pairing, with the pairing potentials $\Delta_{\mathrm{t}}$ and $\Delta_{\mathrm{s}}$, respectively, and also as the chemical potential $\mu$ is varied. The stability of a singly degenerate zero mode is guaranteed in a system with particle-hole symmetry in which the zero mode is isolated from the continuous spectrum by a finite energy gap. Therefore, studying gap-closing surfaces in the parameter space of the coupling constants characterizing the theory is of crucial importance in identifying the stability of the Majorana modes as well as the phase boundaries between different topological phases.

In this paper, we compute the conditions for the closing of the gap in $\Delta_{\mathrm{t}} / \Delta_{\mathrm{s}}-\mu$ space by exploring a one-to-one mapping to the normal-state dispersion relation, in which a function of the ratio $\Delta_{\mathrm{t}} / \Delta_{\mathrm{s}}$ serves as a reparameterization of the magnitude of the momenta in the dispersion relation. Thereto, we show that there are as many lines in $\Delta_{\mathrm{t}} / \Delta_{\mathrm{s}}-\mu$ space at which the gap closes as there are branches in the dispersion relation. But in the case of the Rashba-Dirac dispersion, it is possible to go from one side of a gap vanishing line to another without crossing it by going through the point at infinity $\left(\Delta_{\mathrm{s}}=0\right)$. Thus, there are not two distinct phases separated by a transition in this case but there is a single phase instead.

In the Fermi limit relevant to 2D TRS noncentrosymmetric superconductors, we find that the conditions for the closing of the spectral gap do separate two gapful phases. These two regimes are those in which either the singlet or the triplet pairing controls the physics. The detailed shape of the phase boundaries is dictated by the normal-state dispersion relation. The presence of the TRS spin-orbit coupling leads to interesting effects at certain values of the chemical potential, for example, re-entrance to the phase dominated by singletpairing physics even when $\Delta_{\mathrm{t}} / \Delta_{\mathrm{s}}$ is large. We find two Majorana zero modes bound to an isolated vortex in the triplet controlled phase but they disappear in the singlet controlled phase. We find that the vortices that bind this pair of Majorana zero modes have unit flux, as opposed to the half vortices needed in the case of two-dimensional $p_{x} \pm i p_{y}$ superconductors that break time-reversal symmetry. The physical reason for this difference is that, when TRS holds, the spinresolved pairing amplitudes $\Delta_{\uparrow \uparrow}$ and $\Delta_{\downarrow \downarrow}$ are not independent, and thus one cannot introduce vorticity in one but not the other, which is the case for the half vortices in the $p_{x} \pm i p_{y}$ superconductors. Therefore the pair of Majorana fermions that we find for full vortices in the triplet case is distinct from those found by Read and Green ${ }^{42}$ and Ivanov. ${ }^{43}$ The pair of Majorana fermions that we find is not robust to a generic weak perturbation that breaks translation invariance, for these Majorana fermions are not related to each other by the operation of time reversal. This pair of Majorana fermions is thus unrelated to the one introduced by Qi et al. in Ref. 32 as a mean to identify the triplet dominated TRS phase as a nontrivial $2 \mathrm{D} \mathbb{Z}_{2}$ topological superconducting phase. We conclude that, although both superconducting regimes can be distinguished by the even number of Majorana fermions that an isolated TRS-breaking vortex binds, this distinction is not topological, for it is not robust to static disorder, for example.

In addition to this interesting interplay between the singlet and triplet pair potentials for the existence of Majorana fer- 
mions, superconductivity for surface states in a 3D TRS topological band insulator and noncentrosymmetric materials has other curious properties, one of which is the following. Because spin is not a good quantum number, even if the pair potential contains, say, only the singlet component, the condensate will nevertheless have triplet correlations. For example, the pairing correlation for electrons $\left\langle c_{\boldsymbol{k} \uparrow} c_{-\boldsymbol{k} \uparrow}\right\rangle \neq 0$ (and $\left\langle c_{\boldsymbol{k} \downarrow} c_{-\boldsymbol{k} \downarrow}\right\rangle \neq 0$ as well) for $\mu \neq 0$, even if $\Delta_{\mathrm{t}}=0$. One measurable consequence is that, generically, these superconducting states would lead to detectable Josephson currents when connected to either conventional $s$-wave or $p$-wave superconductors, manifesting the fact that they have both types of correlations (even if only the singlet pair potential $\Delta_{\mathrm{s}}$ is nonzero).

The paper is organized as follows. We define the model in Sec. II. We show in Sec. III that the normal-state Pauli magnetic susceptibility has the remarkable property that it depends on all states in the Fermi sea, not only on the density of states at the Fermi level, and that it is anisotropic for the surface states of a 3D TRS topological band insulator. The dynamical Pauli susceptibility tensor in both the normal and superconducting states encodes rich magnetoelectric effects that are responsible for the spin-Hall effect among others. We study in Sec. IV self-consistently the interplay between the singlet and triplet components to the superconducting instabilities of the surface states in a 3D TRS topological band insulator or in 2D noncentrosymmetric materials. The generic mean-field phase diagram for a TRS two-band BdG Hamiltonian in the isotropic continuum limit that interpolates between the regimes (1.1) and (1.2) is constructed in Sec. V. We find that the Rashba-Dirac limit $t / \alpha=0$, that pertains to the surface states of a 3D TRS topological band insulator, is singular in that there exists only one single phase in the phase diagram. In Sec. VI A, we construct explicitly the single Majorana fermion bound to the core of an isolated vortex in the superconducting phase of the surface states of a 3D TRS topological band insulator. In Sec. VI B, we construct explicitly the pair of Majorana fermions bound to the core of an isolated vortex in the triplet dominated superconducting phase of a 2D noncentrosymmetric superconductor. We conclude with Sec. VII.

\section{DEFINITION}

\section{A. Normal state}

In the continuum limit and in the single-particle approximation, we define the single-node Rashba-Dirac Hamiltonian

$$
\mathcal{H}_{0 \boldsymbol{k}}^{\text {sur }}:=\hbar v_{\mathrm{RD}}\left(k_{1} \sigma_{2}-k_{2} \sigma_{1}\right), \quad \hbar v_{\mathrm{RD}}|\boldsymbol{k}|<\Lambda .
$$

The Rashba-Dirac velocity is $v_{\mathrm{RD}}$ and we restrict the momentum $\hbar \boldsymbol{k}$ by the cutoff $\Lambda / v_{\mathrm{RD}}$ beyond which the surface states of a TRS topological band insulator merge into the bulk states. The two-dimensional momentum $\hbar \boldsymbol{k}=\hbar\left(k_{1}, k_{2}\right)$ couples to the Pauli matrices $\boldsymbol{\sigma}=\left(\sigma_{1}, \sigma_{2}\right)$. These Pauli matrices act on the internal space of the spin-1/2 degrees of freedom carried by the surface electron (hole) in the laboratory frame of reference. This coupling between the electron (hole) crystal wave vector and the spin of the electron (hole) pre-

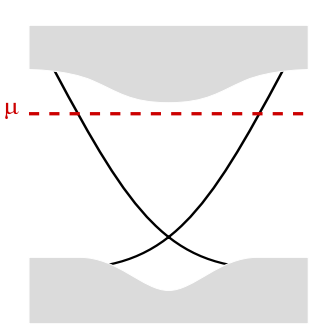

(a)

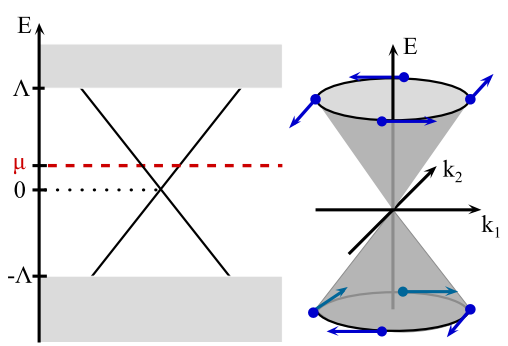

(b)
FIG. 1. (Color online) (a) Schematic picture of the surface states of the topological insulator $\mathrm{Bi}_{2} \mathrm{Se}_{3}$ (black lines). The chemical potential $\mu$ is far from the Rashba-Dirac (nodal) point and close to the conduction-band continuum (upper gray region) while the RashbaDirac (nodal) point is close to the valence-band continuum (lower gray region). (b) Left: one-dimensional cut of the dispersion of the Rashba-Dirac model defined by Eq. (2.1). In particular, we study the case where $\mu$ is close to the Rashba-Dirac (nodal) point rather than close to the energy cutoff $\pm \Lambda$ that defines the onset of the conduction band and the valence band. Right: the expectation values of the electron spins are perpendicular to their momenta and oriented in opposite directions for the upper and lower cone [see Eqs. (3.3b) and (3.3c) at $B=0]$.

vents conservation of the electron (hole) spin. However, TRS is conserved so that the linear dispersion that follows from Eq. (2.1) is twofold Kramers degenerate.

For the surface states of $\mathrm{Bi}_{2} \mathrm{Se}_{3}$, the Rashba-Dirac velocity is measured to be $v_{\mathrm{RD}} \approx 5.1 \times 10^{5} \mathrm{~m} \mathrm{~s}^{-1}$. ${ }^{3}$ Furthermore, the Rashba-Dirac energy (the energy measured at the Rashba-Dirac point) $\varepsilon_{\mathrm{RD}} \approx 0.3 \mathrm{eV}$ is close to the insulating band gap of $0.35 \mathrm{eV}$ for the bulk states in which the surface states merge. ${ }^{3}$ Hence, the superconducting gap $\Delta \approx 3$ $\times 10^{-4} \mathrm{eV}$ in intercalated $\mathrm{Cu}_{x} \mathrm{Bi}_{2} \mathrm{Se}_{3}$ is minute compared to $\varepsilon_{\mathrm{RD}}$ in $\mathrm{Bi}_{2} \mathrm{Se}_{3}$. If there are Rashba-Dirac surface states in $\mathrm{Cu}_{x} \mathrm{Bi}_{2} \mathrm{Se}_{3}$ involved in pairing correlations or if there are Rashba-Dirac surface states in $\mathrm{Bi}_{2} \mathrm{Se}_{3}$ involved in pairing correlations induced by the proximity to superconducting $\mathrm{Cu}_{x} \mathrm{Bi}_{2} \mathrm{Se}_{3}$, they are likely to be far away from the RashbaDirac point. On the other hand, we take the point of view that it is only a matter of time before a way is found to tune the chemical potential of the TRS topological surface states through the Rashba-Dirac point (substituted $\mathrm{Cu}_{y} \mathrm{Bi}_{2-y} \mathrm{Se}_{3}$ might be a candidate). Hence, one goal of this paper is to characterize pairing correlations among the surface states of a TRS topological band insulator upon tuning the Fermi energy through the Rashba-Dirac point (see Fig. 1).

We shall compare our study of Eq. (2.1) with that of the two-dimensional Rashba tight-binding model

$$
\mathcal{H}_{0 \boldsymbol{k}}^{2 \mathrm{D}}:=\varepsilon_{k} \sigma_{0}+\boldsymbol{g}_{\boldsymbol{k}} \cdot \boldsymbol{\sigma}, \quad \boldsymbol{k} \in \mathrm{BZ} .
$$

Here, $\sigma_{0}$ is the unit $2 \times 2$ matrix in spin space and the wave vector $\boldsymbol{k}$ is restricted to the first Brillouin zone (BZ). It describes the hopping on a square lattice with the SRS dispersion

$$
\varepsilon_{k}=-2 t\left(\cos k_{1}+\cos k_{2}\right), \quad t \geq 0
$$

and with the Rashba spin-orbit coupling 


$$
\boldsymbol{g}_{k}=\alpha\left(\begin{array}{c}
-\sin k_{2} \\
\sin k_{1}
\end{array}\right),
$$

say. Our convention throughout this paper will be that $\alpha$ $\geq 0$.

An important difference between the surface states of a 3D TRS topological band insulator and the Rashba tightbinding states is that the surface states span an odd number less of Fermi surfaces. This is a manifestation of the fermion doubling that occurs when attempting to regularize a $D$-dimensional single Rashba-Dirac cone by a $D$-dimensional tight-binding model. The fermion doubling can only be eliminated by the addition of the Wilson term

$$
\mathcal{H}_{0 k}^{\mathrm{W}}:=t_{\mathrm{W}}\left(2-\cos k_{1}-\cos k_{2}\right) \sigma_{3}, \quad t_{\mathrm{W}} \gg t+\alpha,
$$

to the tight-binding Hamiltonian (2.2a) at the cost of breaking TRS.

Hamiltonian (2.1) is scale invariant. It then follows that the density of states per unit area is proportional to the absolute value of the chemical potential $\mu$ and vanishes at the Rashba-Dirac point $\mu=0$. The effects of this scale invariance on charge transport, including the orbital effects of a magnetic field, are identical to those in graphene in the single Rashba-Dirac cone approximation, if the Zeeman coupling to a magnetic field is ignored. In Sec. III, we are going to study the inherently strong effects of the spin-orbit coupling on the Pauli magnetic susceptibility. However, before doing so, we want to include the possibility of a superconducting instability that we first treat at the mean-field level without imposing the condition of self-consistency.

\section{B. Mean-field superconducting state}

We rewrite the continuum Hamiltonian (2.1) or the lattice Hamiltonian (2.2a) in the language of second quantization. For simplicity, we choose a tight-binding notation. Reverting notation to the continuum is straightforward. We thus introduce the spinor $\psi_{\boldsymbol{k}}^{\dagger}=\left(c_{\boldsymbol{k} \uparrow}^{\dagger}, c_{\boldsymbol{k} \downarrow}^{\dagger}\right)$ for electrons in the spin basis of the laboratory frame of reference and the spinor $\tilde{\psi}_{k}^{\dagger}$ $=\left(a_{k_{+}}^{\dagger}, a_{k_{-}}^{\dagger}\right)$ in the helicity basis defined below. This gives

$$
\begin{gathered}
H_{0}=\sum_{k \in \mathrm{BZ}} \psi_{\boldsymbol{k}}^{\dagger} \mathcal{H}_{0 ; k} \psi_{k}=\sum_{k \in \mathrm{BZ}} \tilde{\psi}_{\boldsymbol{k}}^{\dagger} \tilde{\mathcal{H}}_{0 ; k} \tilde{\psi}_{k}, \\
\mathcal{H}_{0 ; k}=\left(\varepsilon_{k}-\mu\right) \sigma_{0}+\boldsymbol{g}_{\boldsymbol{k}} \cdot \boldsymbol{\sigma}, \\
\tilde{\mathcal{H}}_{0 ; \boldsymbol{k}}=\left(\begin{array}{cc}
\xi_{k+} & 0 \\
0 & \xi_{k-}
\end{array}\right) .
\end{gathered}
$$

The single-particle dispersion is here given by ${ }^{50}$

$$
\xi_{k \pm}=\varepsilon_{k}-\mu \pm\left|g_{k}\right|
$$

while the transformation between the laboratory basis and the helicity basis is given by the unitary $2 \times 2$ matrix

$$
\Pi_{k} \equiv \frac{1}{\sqrt{2}}\left(\begin{array}{cc}
1 & 1 \\
e^{i \varphi_{k}} & -e^{i \varphi_{k}}
\end{array}\right):=\frac{1}{\sqrt{2}}\left(\begin{array}{cc}
1 & 1 \\
\frac{g_{k 1}+i g_{k 2}}{\left|g_{k}\right|} & -\frac{g_{k 1}+i g_{k 2}}{\left|g_{k}\right|}
\end{array}\right),
$$

whereby

$$
\psi_{k}^{\dagger}=\tilde{\psi}_{k}^{\dagger} \Pi_{k}^{\dagger}, \quad \psi_{k}=\Pi_{k} \tilde{\psi}_{k}, \quad \tilde{\mathcal{H}}_{0 ; k}=\Pi_{k}^{\dagger} \mathcal{H}_{0 ; k} \Pi_{k} .
$$

The (mean-field) BdG Hamiltonian is defined by

$$
\begin{aligned}
H & :=\sum_{k \in \mathrm{BZ}} \Psi_{k}^{\dagger}\left(\begin{array}{cc}
\mathcal{H}_{0 ; k} & \Delta_{k} \\
\Delta_{k}^{\dagger} & -\mathcal{H}_{0 ;-k}^{T}
\end{array}\right) \Psi_{k} \\
& =\sum_{k \in \mathrm{BZ}} \Phi_{k}^{\dagger}\left(\begin{array}{cc}
\mathcal{H}_{0 ; k} & \Delta_{k}\left(-i \sigma_{2}\right) \\
i \sigma_{2} \Delta_{k}^{\dagger} & -\sigma_{2} \mathcal{H}_{0 ;-k}^{T} \sigma_{2}
\end{array}\right) \Phi_{k} \\
& =\sum_{k \in \mathrm{BZ}} \tilde{\Phi}_{k}^{\dagger}\left(\begin{array}{cc}
\tilde{\mathcal{H}}_{0 ; k} & \tilde{\Delta}_{k} \\
\tilde{\Delta}_{\boldsymbol{k}}^{\dagger} & -\tilde{\mathcal{H}}_{0 ;-k}^{T}
\end{array}\right) \tilde{\Phi}_{k},
\end{aligned}
$$

where the bispinors $\Psi_{k}^{\dagger}, \Phi_{k}^{\dagger}$, and $\tilde{\Phi}_{k}^{\dagger}$ are given by

$$
\begin{gathered}
\Psi_{k}^{\dagger}=\left(\psi_{k}^{\dagger}, \psi_{-k}\right)=\left(c_{\boldsymbol{k} \uparrow}^{\dagger}, c_{\boldsymbol{k} \downarrow}^{\dagger}, c_{-\boldsymbol{k} \uparrow}, c_{-\boldsymbol{k} \downarrow}\right), \\
\Phi_{\boldsymbol{k}}^{\dagger}=\left(\psi_{k}^{\dagger}, i \sigma_{2} \psi_{-k}\right)=\left(c_{\boldsymbol{k} \uparrow}^{\dagger}, c_{\boldsymbol{k} \downarrow}^{\dagger}, c_{-k \downarrow},-c_{-\boldsymbol{k} \uparrow}\right), \\
\tilde{\Phi}_{\boldsymbol{k}}^{\dagger}=\left(a_{k+}^{\dagger}, a_{k-}^{\dagger}, e^{i \varphi_{-k}} a_{-k+},-e^{i \varphi_{-k}} a_{-k-}\right),
\end{gathered}
$$

respectively. We have chosen to construct the bispinors $\Phi_{k}^{\dagger}$ and $\widetilde{\Phi}_{k}^{\dagger}$ from the spinors $\psi_{k}^{\dagger}$ and $\tilde{\psi}_{k}^{\dagger}$, respectively, and their time-reversed partners. Thereby, we have to take care of the action of the time-reversal operation $\mathcal{T}$ on the laboratory and the helicity single-particle states labeled by the wave vector $\boldsymbol{k}$ and the indices $s=\uparrow, \downarrow$ and $\lambda= \pm 1$, respectively. For the laboratory basis, it is

$$
\mathcal{T}|\boldsymbol{k} \uparrow\rangle=+|-\boldsymbol{k} \downarrow\rangle, \quad \mathcal{T}|\boldsymbol{k} \downarrow\rangle=-|-\boldsymbol{k} \uparrow\rangle,
$$

i.e., it is off-diagonal in the laboratory spin basis. For the helicity basis,

$$
\begin{aligned}
& |\boldsymbol{k}+\rangle=\frac{1}{\sqrt{2}}\left(|\boldsymbol{k} \uparrow\rangle+e^{+i \varphi_{k}}|\boldsymbol{k} \downarrow\rangle\right), \\
& |\boldsymbol{k}-\rangle=\frac{1}{\sqrt{2}}\left(|\boldsymbol{k} \uparrow\rangle-e^{+i \varphi_{k}}|\boldsymbol{k} \downarrow\rangle\right),
\end{aligned}
$$

together with

$$
e^{i \varphi_{k}}=-e^{i \varphi_{-k}}
$$

imply that it is

$$
\mathcal{T}|\boldsymbol{k} \lambda\rangle=\lambda e^{-i \varphi_{-}}|-\boldsymbol{k} \lambda\rangle,
$$

i.e., it is diagonal in the helicity internal space but with the wave vector and helicity-dependent eigenvalue $\lambda e^{i \varphi_{k}}$ that is odd under the inversion $\boldsymbol{k} \rightarrow-\boldsymbol{k}$. Hence, the bispinors $\Phi_{\boldsymbol{k}}^{\dagger}$ and $\tilde{\Phi}_{k}^{\dagger}$ follow. 
We parameterize the $2 \times 2$ pair-potential matrix by

$$
\Delta_{k}=\left(\Delta_{\mathrm{s}, \boldsymbol{k}} \sigma_{0}+\boldsymbol{d}_{\boldsymbol{k}} \cdot \boldsymbol{\sigma}\right)\left(i \sigma_{2}\right)
$$

in the laboratory frame for the spin degrees of freedom. PHS, which embodies Fermi statistics within the BdG formulation, demands that it is an antisymmetric operator, i.e.,

$$
\Delta_{\mathrm{s}, k}=\Delta_{\mathrm{s},-k}, \quad d_{k}=-d_{-k} .
$$

TRS imposes the conditions

$$
\Delta_{\mathrm{s}, k}=\Delta_{\mathrm{s},-k}^{*}, \quad d_{k}=-d_{-k}^{*} .
$$

Throughout this paper, we consider Cooper pairs made of time-reversed helicity single-particle states from Eq. (2.7c). Hence, we take the $2 \times 2$ pair-potential matrix

$$
\widetilde{\Delta}_{k}=\left(\begin{array}{cc}
\widetilde{\Delta}_{k+} & 0 \\
0 & \widetilde{\Delta}_{k-}
\end{array}\right)
$$

to be diagonal in the helicity basis, and it then follows that

$$
\widetilde{\Delta}_{k+}=\widetilde{\Delta}_{-k+}^{*}, \quad \widetilde{\Delta}_{k-}=\widetilde{\Delta}_{-k-}^{*},
$$

as a consequence of TRS. Furthermore, we find with the help of Eq. (2.5b) the $4 \times 4$ Hermitian matrix (the complex notation $z=x+i y$ and $\bar{z}=x-i y$ is occasionally used)

$$
\mathcal{H}_{k}=\left(\begin{array}{cccc}
\varepsilon_{k}-\mu & \bar{g}_{k} & \Delta_{\mathrm{s}, k} & \Delta_{\mathrm{t}, \boldsymbol{k}} e^{-i \varphi_{k}} \\
g_{k} & \varepsilon_{k}-\mu & \Delta_{\mathrm{t}, \boldsymbol{k}} e^{+i \varphi_{k}} & \Delta_{\mathrm{s}, \boldsymbol{k}} \\
\text { c.c. } & \text { c.c. } & -\varepsilon_{k}+\mu & -\bar{g}_{k} \\
\text { c.c. } & \text { c.c. } & -g_{k} & -\varepsilon_{k}+\mu
\end{array}\right),
$$

where we recall that $\varphi_{k}:=\arg g_{k}$ and

$$
\begin{gathered}
\Delta_{\mathrm{s}, k}=\frac{1}{2}\left(\widetilde{\Delta}_{k+}+\widetilde{\Delta}_{k-}\right)=\Delta_{\mathrm{s},-k}^{*}, \\
\Delta_{\mathrm{t}, k}=\frac{1}{2}\left(\widetilde{\Delta}_{k+}-\widetilde{\Delta}_{k-}\right)=\Delta_{\mathrm{t},-k}^{*}, \\
\boldsymbol{d}_{k}=\frac{1}{2}\left(\tilde{\Delta}_{k+}-\tilde{\Delta}_{k-}\right) \frac{\boldsymbol{g}_{k}}{\left|\boldsymbol{g}_{k}\right|}=-\boldsymbol{d}_{-k}^{*},
\end{gathered}
$$

in the $\Phi_{\boldsymbol{k}}$ representation of Eq. (2.5a).

The fact that the vector $\boldsymbol{d}_{\boldsymbol{k}}$ is parallel to $\boldsymbol{g}_{\boldsymbol{k}}$ is a consequence of our assumption that Cooper pairs are made of time-reversed helicity single-particle states, i.e., Eq. (2.9a). This assumption is justified if the pairing interaction preserves the symmetry of the noninteracting Hamiltonian. Following the literature on noncentrosymmetric superconductors, ${ }^{22}$ we are thus assuming that the symmetry of the noninteracting Hamiltonian is preserved by the selfconsistent inclusion of the pairing interaction.

We also demand that Hamiltonian (2.10a) is single valued in the BZ. This restricts the triplet pairing $\Delta_{\mathrm{t}, \boldsymbol{k}}$ to vanish at least as fast as $\left|\boldsymbol{g}_{\boldsymbol{k}}\right|$,

$$
\lim _{\left|\boldsymbol{g}_{\boldsymbol{k}}\right| \rightarrow 0} \frac{\left|\Delta_{\mathrm{t}, \boldsymbol{k}}\right|}{\left|\boldsymbol{g}_{\boldsymbol{k}}\right|}<c
$$

for some number $c$ larger than or equal to 0 . With our choice of gauge, $\widetilde{\Delta}_{k+}$ and $\widetilde{\Delta}_{k-}$ or, equivalently, $\Delta_{\mathrm{s}, \boldsymbol{k}}$ and $\Delta_{\mathrm{t}, \boldsymbol{k}}$ are real valued. In Sec. VI, where we study TRS-breaking vortices, we revert instead to complex order parameters to accommodate twists in the phases of the singlet and triplet pair potentials. Finally, we observe that the pair-potential eigenvalues

$$
\tilde{\Delta}_{k \lambda}=\Delta_{\mathrm{s}, k}+\lambda d_{k} \cdot \frac{g_{k}}{\left|g_{k}\right|}, \quad \lambda= \pm,
$$

transform according to the same irreducible representation of the space group. For example, in the isotropic continuum limit with $s$-wave pairing they are functions of $|\boldsymbol{k}|$ only.

The BdG Hamiltonian (2.5a) is of the form

$$
H \equiv \sum_{\lambda= \pm} H_{\lambda}:=\sum_{\lambda= \pm} \sum_{k \in \mathrm{BZ}} H_{k \lambda},
$$

$$
H_{k \lambda}=\xi_{k \lambda} a_{k \lambda}^{\dagger} a_{k \lambda}+\lambda \widetilde{\Delta}_{k \lambda}\left(e^{i \varphi_{-k}} a_{-k \lambda} a_{k \lambda}+\text { H.c. }\right)
$$

The mean-field ground state is the state $\left|Y_{\text {mf }}\right\rangle$ annihilated by $H$. It is obtained as the direct product $\left|Y_{\mathrm{mf}}\right\rangle=\left|Y_{\mathrm{mf}}^{+}\right\rangle \otimes\left|Y_{\mathrm{mf}}^{-}\right\rangle$, where $\left|Y_{\mathrm{mf}}^{\lambda}\right\rangle$ is annihilated by $H_{\lambda}$ for each of the helicities $\lambda= \pm$.

Each helicity supports quasiparticles obeying the PHS (relative to the chemical potential) dispersion $\pm E_{k \lambda}$ with

$$
E_{k \lambda}=\sqrt{\xi_{k \lambda}^{2}+\widetilde{\Delta}_{k \lambda}^{2}}, \quad \lambda= \pm .
$$

However, the ground states $\left|Y_{\mathrm{mf}}^{+}\right\rangle$and $\left|Y_{\mathrm{mf}}^{-}\right\rangle$are not independent since they are tied to each other by TRS. Indeed, TRS implies that the relative phase of the pairing potentials $\widetilde{\Delta}_{k \lambda}$ with the helicities $\lambda= \pm$ is locked to be 0 or $\pi$, as follows from the transformation law (2.7c), i.e.,

$$
\mathcal{T} a_{k \lambda}^{\dagger} \mathcal{T}^{-1}=\lambda e^{-i \varphi_{k}} a_{-k \lambda}^{\dagger}, \quad \mathcal{T} a_{k \lambda} \mathcal{T}^{-1}=\lambda e^{+i \varphi_{-k}} a_{-k \lambda}
$$

To construct $\left|Y_{\mathrm{mf}}\right\rangle$, we perform a Bogoliubov transformation for each helicity index $\lambda= \pm$ independently. Thus, for each helicity $\lambda= \pm$, we define

$$
\gamma_{k \lambda}:=U_{k \lambda} a_{k \lambda}-V_{k \lambda} a_{-k \lambda}^{\dagger}
$$

with the complex-valued coefficients $U_{k \lambda}$ and $V_{k \lambda}$,

$$
\begin{gathered}
\left|U_{k \lambda}\right|^{2}:=\frac{1}{2}\left(1+\frac{\xi_{k \lambda}}{E_{k \lambda}}\right), \\
\left|V_{k \lambda}\right|^{2}:=\frac{1}{2}\left(1-\frac{\xi_{k \lambda}}{E_{k \lambda}}\right), \\
\frac{U_{k \lambda}}{V_{k \lambda}}=-\frac{\lambda e^{-i \varphi_{-k}} \tilde{\Delta}_{k \lambda}}{E_{k \lambda}-\xi_{k \lambda}} .
\end{gathered}
$$

Under this transformation 


$$
H=\sum_{k \in \mathrm{BZ}} \sum_{\lambda= \pm} E_{k \lambda} \gamma_{k \lambda}^{\dagger} \gamma_{k \lambda} .
$$

The mean-field ground state is then

$$
\left|\Upsilon_{\mathrm{mf}}\right\rangle=\prod_{\lambda= \pm} \prod_{k}\left(U_{k \lambda}+V_{k \lambda} a_{k \lambda}^{\dagger} a_{-k \lambda}^{\dagger}\right)|0\rangle
$$

provided

$$
\gamma_{k \lambda}\left|Y_{\mathrm{mf}}\right\rangle=0
$$

holds for all $\boldsymbol{k}$ and all $\lambda= \pm$.

By construction, the mean-field ground state (2.17) is TRS. SRS is, however, broken. Consequently,

$$
\begin{gathered}
\left\langle\mathrm{Y}_{\mathrm{mf}}\left|c_{-k \uparrow} c_{k \uparrow}\right| Y_{\mathrm{mf}}\right\rangle=\frac{e^{-i \varphi_{-k}}}{4}\left(\frac{\tilde{\Delta}_{k+}}{E_{k+}}-\frac{\tilde{\Delta}_{k-}}{E_{k-}}\right), \\
\left\langle\mathrm{Y}_{\mathrm{mf}}\left|c_{-k \downarrow} c_{k \downarrow}\right| Y_{\mathrm{mf}}\right\rangle=\frac{e^{i \varphi_{k}}}{4}\left(\frac{\widetilde{\Delta}_{k+}}{E_{k+}}-\frac{\tilde{\Delta}_{k-}}{E_{k-}}\right), \\
\left\langle\mathrm{Y}_{\mathrm{mf}}\left|c_{-k \uparrow} c_{k \downarrow}\right| Y_{\mathrm{mf}}\right\rangle=\frac{1}{4}\left(\frac{\tilde{\Delta}_{k+}}{E_{k+}}+\frac{\tilde{\Delta}_{k-}}{E_{k-}}\right),
\end{gathered}
$$

are generically nonvanishing (one exception is the RashbaDirac limit $\varepsilon_{k}=0$ at the Rashba-Dirac point $\mu=0$ ) even though the pair potential may be purely singlet when

$$
\widetilde{\Delta}_{k+}=\widetilde{\Delta}_{k-}
$$

or purely triplet when

$$
\widetilde{\Delta}_{k+}=-\widetilde{\Delta}_{k-} .
$$

In a superconducting state that preserves SRS, the ground state has no spin correlations other than that of the pair condensate.

\section{SUSCEPTIBILITY}

\section{A. Static and uniform Pauli magnetic susceptibility at $T=0$ in the normal state}

We are after the Pauli magnetization per electron induced by the Zeeman coupling $\propto-B_{1} \sigma_{1}-B_{2} \sigma_{2}-B_{3} \sigma_{3}$, where it is understood that $\sigma_{3}$ is the third Pauli matrix and the in-plane components of the magnetic field are $B_{1}$ and $B_{2}$ while the out-of-plane component $B_{3}$ is taken along the spin quantization axis in the laboratory frame of reference.

To obtain the Pauli magnetization per electron at $T=0$, we start from Eq. (2.1) with the Zeeman coupling added

$$
\mathcal{H}_{B k}^{\text {sur }}:=\left(\hbar v_{\mathrm{RD}} k_{1}-B_{2}\right) \sigma_{2}-\left(\hbar v_{\mathrm{RD}} k_{2}+B_{1}\right) \sigma_{1}-B_{3} \sigma_{3},
$$

compute the expectation value of the spin operator $\hbar \sigma_{1,2,3} / 2$ for all the Bloch states, and sum these expectation values up to the chemical potential $\mu$. The Pauli susceptibility per electron then follows by differentiation with respect to $B_{1,2,3}$ followed by setting $B_{1,2,3}=0$. We set $\hbar=v_{\mathrm{RD}}=1$ to simplify notation.
As long as $B_{1}^{2}+B_{2}^{2}>0$, the eigenvalue

$$
\xi_{ \pm}(\boldsymbol{k})=-\mu \pm \sqrt{\left(k_{1}-B_{2}\right)^{2}+\left(k_{2}+B_{1}\right)^{2}+B_{3}^{2}}
$$

has the eigenstate

$$
\Psi_{ \pm}(\boldsymbol{k})=\frac{1}{\mathcal{N}_{ \pm}(\boldsymbol{k})}\left(\begin{array}{c}
-k_{2}-\mathrm{i} k_{1}-B_{1}+\mathrm{i} B_{2} \\
\xi_{ \pm}(\boldsymbol{k})+\mu+B_{3}
\end{array}\right)
$$

with the normalization

$$
\mathcal{N}_{ \pm}(\boldsymbol{k}):=\sqrt{2\left[\xi_{ \pm}(\boldsymbol{k})+\mu\right]\left[\xi_{ \pm}(\boldsymbol{k})+\mu+B_{3}\right]} .
$$

We observe that the effect of in-plane magnetic fields is to translate the Fermi sea. The spin expectation values in the Bloch states are

$$
\begin{gathered}
\Psi_{ \pm}^{\dagger}(\boldsymbol{k}) \sigma_{3} \Psi_{ \pm}(\boldsymbol{k})=\mp \frac{B_{3}}{\sqrt{B_{3}^{2}+|\bar{k}+B|^{2}}} \\
=\mp \frac{B_{3}}{|\boldsymbol{k}|}[F(B / \bar{k}, \bar{B} / k)+\cdots], \quad \\
\left.\Psi_{ \pm}^{\dagger}(\boldsymbol{k}) \sigma_{ \pm} \Psi_{ \pm}(\boldsymbol{k})\right|_{B_{3}=0}=-\frac{2(\bar{k}+B)\left[\xi_{ \pm}(\boldsymbol{k})+\mu\right]}{|\bar{k}+B|^{2}+\left[\xi_{ \pm}(\boldsymbol{k})+\mu\right]^{2}}=\mp \frac{\bar{k}+B}{|\bar{k}+B|} \\
=\mp \frac{\bar{k}+B}{|\boldsymbol{k}|}[F(B / \bar{k}, \bar{B} / k)+\cdots],
\end{gathered}
$$

and

$$
\begin{aligned}
\left.\Psi_{ \pm}^{\dagger}(\boldsymbol{k}) \bar{\sigma} \Psi_{ \pm}(\boldsymbol{k})\right|_{B_{3}=0} & =-\frac{2(k+\bar{B})\left[\xi_{ \pm}(\boldsymbol{k})+\mu\right]}{|\bar{k}+B|^{2}+\left[\xi_{ \pm}(\boldsymbol{k})+\mu\right]^{2}}=\mp \frac{k+\bar{B}}{|k+\bar{B}|} \\
& =\mp \frac{k+\bar{B}}{|\boldsymbol{k}|}[F(\bar{B} / k, B / \bar{k})+\cdots] .
\end{aligned}
$$

Here, we have introduced the complex notations

$$
\sigma=\sigma_{1}+i \sigma_{2}, \quad \bar{\sigma}=\sigma_{1}-i \sigma_{2},
$$

for the Pauli matrices,

$$
k=k_{2}+i k_{1}, \quad \bar{k}=k_{2}-i k_{1},
$$

for the momenta and

$$
B=B_{1}+i B_{2}, \quad \bar{B}=B_{1}-i B_{2},
$$

for the in-plane components of the magnetic field. We have also introduced the real-valued function

$$
F(z, \bar{z}):=1-\frac{1}{2}(z+\bar{z})
$$

that comes about to first order in an expansion in powers of the components of the magnetic field. The magnetization per electron is obtained by integrating over all single-particle energies up to the chemical potential $\mu$. We conclude that the Pauli magnetic susceptibility tensor per electron is 


$$
\chi_{a b} \propto \delta_{a b}\left\{\begin{array}{c}
\pi(\Lambda-|\mu|) \quad \text { if } a=1,2, \\
2 \pi(\Lambda-|\mu|) \quad \text { if } a=3,
\end{array}\right.
$$

in the noninteracting approximation and at $T=0$.

The Pauli magnetic susceptibility per electron (3.5) also holds for the Rashba tight-binding Hamiltonian (2.2a) with minor modifications provided the limit $t / \alpha \rightarrow 0$ is taken, $(N$ is the number of lattice sites)

$$
\chi_{a b}=\delta_{a b}\left\{\begin{array}{l}
\frac{1 / 2}{N} \sum_{|\mu|<\left|g_{k}\right|} \frac{1}{\left|\boldsymbol{g}_{k}\right|} \quad \text { if } a=1,2, \\
\frac{1}{N_{|\mu|<\left|g_{k}\right|}} \frac{1}{\left|\boldsymbol{g}_{k}\right|} \quad \text { if } a=3 .
\end{array}\right.
$$

In the opposite limit $\alpha / t \rightarrow 0$, we cannot use the lattice counterpart to Eq. (3.3a) to compute $\chi_{33}$, since our choice for the spinor representation is singular in this limit. We can however use the lattice counterparts to Eqs. (3.3b) and (3.3c) to compute $\chi_{11}$ and $\chi_{22}$. By isotropy, we then recover the conventional Pauli magnetic susceptibility

$$
\chi_{a b} \propto \delta_{a b} \nu_{\mathrm{F}}(\mu),
$$

where $\nu_{\mathrm{F}}(\mu)$ is the density of states per electron and per spin of the dispersion $\varepsilon_{k}$. This result remains true to first order in $\alpha / t$.

\section{B. Dynamical Pauli susceptibility in the superconducting state}

Another remarkable consequence of the spin-orbit coupling is that charge-density and spin-density fluctuations are coupled, both in the normal and in the superconducting state. ${ }^{17,51-53}$ The spin-Hall effect is a consequence of this coupling. ${ }^{51,52}$ To quantify this statement, we introduce the susceptibility tensor in the superconducting state

$$
\begin{aligned}
& \left(\hat{\chi}_{00}\right)_{q}=-\frac{1}{\beta N} \sum_{k} \operatorname{tr}\left[G_{0 ; k} X_{03} G_{0 ; k+q} X_{03}\right], \\
& \left(\hat{\chi}_{0 d}\right)_{q}=-\frac{1}{\beta N} \sum_{k} \operatorname{tr}\left[G_{0 ; k} X_{03} G_{0 ; k+q} X_{d 0}\right], \\
& \left(\hat{\chi}_{b 0}\right)_{q}=-\frac{1}{\beta N} \sum_{k} \operatorname{tr}\left[G_{0 ; k} X_{b 0} G_{0 ; k+q} X_{03}\right], \\
& \left(\hat{\chi}_{b d}\right)_{q}=-\frac{1}{\beta N} \sum_{k} \operatorname{tr}\left[G_{0 ; k} X_{b 0} G_{0 ; k+q} X_{d 0}\right],
\end{aligned}
$$

where the indices $b$ and $d$ run over the values 1,2 , and 3 , and

$$
X_{\mu \nu}:=\sigma_{\mu} \otimes \tau_{\nu}, \quad \mu, \nu=0,1,2,3
$$

with the unit $2 \times 2$ matrix $\tau_{0}$ and the Pauli matrices $\tau$ acting on the particle-hole two-dimensional subspace. According to the mean-field Hamiltonian in the superconducting state (2.5a) the single-particle Green's function is

$$
\begin{aligned}
G_{0 ; k}:= & {\left[-i \omega_{n} X_{00}+\left(\varepsilon_{k}-\mu\right) X_{03}+g_{k 1} X_{13}+g_{k 2} X_{23}\right.} \\
& \left.+\Delta_{s, k} X_{01}+\Delta_{t, k}\left(\hat{g}_{k 1} X_{11}+\hat{g}_{k 2} X_{21}\right)\right]^{-1} .
\end{aligned}
$$

Our notation applies to a lattice made of $N$ sites, periodic boundary conditions are assumed, $\beta$ is the inverse temperature (the Boltzmann constant is set to unity), finally $q$ $=\left(i \varpi_{l}, \boldsymbol{q}\right)$ and $k=\left(i \omega_{n}, \boldsymbol{k}\right)$ are three vectors with bosonic and fermionic Matsubara frequencies, respectively, while $\boldsymbol{q}$ and $\boldsymbol{k}$ belong to the first BZ. It is straightforward to modify this notation for the case of the continuum limit.

After performing the summation over the fermionic Matsubara frequencies in Eq. (3.8a) and with some additional lengthy algebra, the dynamical Pauli susceptibility tensor simplifies to

$$
\begin{aligned}
\left(\hat{\chi}_{\mu \nu}\right)_{q}= & \frac{1}{4 N} \sum_{k} \sum_{\lambda, \lambda^{\prime}, \gamma, \gamma^{\prime}}\left(\Gamma_{\mu \nu}^{\lambda, \lambda^{\prime}}\right)_{k, q}\left(C_{\mu \nu}^{\lambda, \lambda^{\prime}, \gamma, \gamma^{\prime}}\right)_{k, q} \\
& \times \frac{f_{\mathrm{FD}}\left(\gamma E_{k \lambda}\right)-f_{\mathrm{FD}}\left(\gamma^{\prime} E_{k+q \lambda^{\prime}}\right)}{\gamma E_{k \lambda}-\gamma^{\prime} E_{k+q \lambda^{\prime}}+i \varpi_{l}},
\end{aligned}
$$

where $\gamma, \gamma^{\prime}= \pm$ and $\mu, \nu=0,1,2,3$, the single-particle dispersion in the superconducting state $E_{k \lambda}$ is defined in Eq. (2.13) for the helicities $\lambda= \pm$, while

$$
f_{\mathrm{FD}}(z)=\frac{1}{e^{\beta z}+1}
$$

is the Fermi-Dirac function. The vertex is given by $\left(\hat{g}_{k}\right.$ $\left.\equiv g_{k} /\left|g_{k}\right|\right)$,

$$
\begin{gathered}
\left(\Gamma_{00}^{\lambda, \lambda^{\prime}}\right)_{\boldsymbol{k}, \boldsymbol{q}}=1+\lambda \lambda^{\prime} \hat{\boldsymbol{g}}_{\boldsymbol{k}} \cdot \hat{\boldsymbol{g}}_{\boldsymbol{k}+\boldsymbol{q}}, \\
\left(\Gamma_{b 0}^{\lambda, \lambda^{\prime}}\right)_{\boldsymbol{k}, \boldsymbol{q}}=\lambda^{\prime} \hat{g}_{b ; \boldsymbol{k}+\boldsymbol{q}}+\lambda \hat{g}_{b ; \boldsymbol{k}}+\lambda \lambda^{\prime} i \epsilon_{a b c} \hat{g}_{a ; \boldsymbol{k}} \hat{g}_{c ; \boldsymbol{k}+q}, \\
\left(\Gamma_{0 d}^{\lambda, \lambda^{\prime}}\right)_{k, q}=\lambda^{\prime} \hat{g}_{d ; \boldsymbol{k}+\boldsymbol{q}}+\lambda \hat{g}_{d ; \boldsymbol{k}}-\lambda \lambda^{\prime} i \epsilon_{a d c} \hat{g}_{a ; \boldsymbol{k}} \hat{g}_{c ; \boldsymbol{k}+q}, \\
\left(\Gamma_{b d}^{\lambda, \lambda^{\prime}}\right)_{\boldsymbol{k}, \boldsymbol{q}}=\delta_{b d}+\lambda \lambda^{\prime} \hat{g}_{a ; k} f_{b d}^{a c} \hat{g}_{c ; \boldsymbol{k}+\boldsymbol{q}}+i \epsilon_{a b d}\left(\lambda \hat{g}_{a ; \boldsymbol{k}}-\lambda^{\prime} \hat{g}_{a ; \boldsymbol{k}+\boldsymbol{q}}\right),
\end{gathered}
$$

where the tensor $f_{b d}^{a c}$ is defined by

$$
f_{b d}^{a c}:=\delta_{a b} \delta_{c d}-\delta_{a c} \delta_{b d}+\delta_{a d} \delta_{b c}=f_{d b}^{a c} .
$$

Finally, the coherence factors are given by

$$
\begin{gathered}
\left(C_{00}^{\lambda, \lambda^{\prime}, \gamma, \gamma^{\prime}}\right)_{k, q}=1+\gamma \gamma^{\prime} \frac{\xi_{k, \lambda} \xi_{k+q, \lambda^{\prime}}-\widetilde{\Delta}_{k, \lambda} \widetilde{\Delta}_{k+q, \lambda^{\prime}}}{E_{k+q, \lambda^{\prime}} E_{k, \lambda}}, \\
\left(C_{b 0}^{\lambda, \lambda^{\prime}, \gamma, \gamma^{\prime}}\right)_{k, q}=\left(C_{0 d}^{\lambda, \lambda^{\prime}, \gamma, \gamma^{\prime}}\right)_{k, q}=\gamma \frac{\xi_{k, \lambda}}{E_{k, \lambda}}+\gamma^{\prime} \frac{\xi_{k+q, \lambda^{\prime}}}{E_{k+q, \lambda^{\prime}}}, \\
\left(C_{b d}^{\lambda, \lambda^{\prime}, \gamma, \gamma^{\prime}}\right)_{k, q}=1+\gamma \gamma^{\prime} \frac{\xi_{k, \lambda} \xi_{k+q, \lambda^{\prime}}+\widetilde{\Delta}_{k, \lambda} \widetilde{\Delta}_{k+q, \lambda^{\prime}}}{E_{k+q, \lambda^{\prime}} E_{k, \lambda}},
\end{gathered}
$$

where the single-particle dispersion in the normal state $\xi_{k, \lambda}$ and the superconducting pair potentials $\widetilde{\Delta}_{k, \lambda}$ are defined in Eq. (2.4b) and in Eq. (2.9a), respectively. The dynamical Pauli susceptibility of the normal state is obtained by taking the limit $\widetilde{\Delta}_{k, \lambda} \rightarrow 0, \lambda= \pm$, supplemented by the replacements 


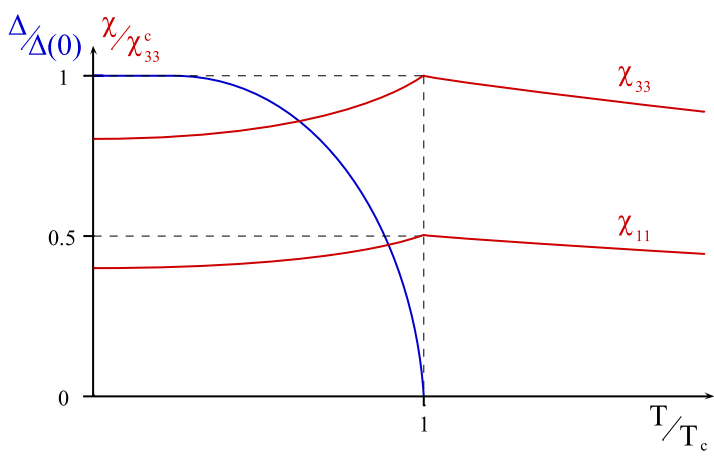

FIG. 2. (Color online) Temperature dependence of the selfconsistent superconducting gap (red, normalized by the value at zero temperature) and the in-plane and out-of-plane Pauli magnetic susceptibility (red, normalized by the maximum of the out-of-plane susceptibility) for $\mu=5 \omega_{\mathrm{D}}$ and $|V| \nu\left(\omega_{\mathrm{D}}\right)=1$. Here, $\omega_{\mathrm{D}}$ is the Debye cutoff used for the gap equations.

$\gamma E_{k \lambda} \rightarrow E_{k \lambda}$ and $\gamma^{\prime} E_{k+q \lambda^{\prime}} \rightarrow E_{k+q \lambda^{\prime}}$ so as to remove the particle-hole symmetry.

We then recover the result from Ref. 54. If we furthermore set $\varpi_{l}=0$ in Eq. (3.9a), we obtain the static susceptibility of the normal state. At the Rashba-Dirac point, i.e., for $\xi_{k \lambda}=\lambda\left|g_{k}\right|$, the following components of the static susceptibility vanish: $\hat{\chi}_{01}, \hat{\chi}_{02}, \hat{\chi}_{10}, \hat{\chi}_{20}, \hat{\chi}_{13}, \hat{\chi}_{23}, \hat{\chi}_{31}$, and $\hat{\chi}_{32}$.

We now consider the isotropic continuum limit (2.1) (the Rashba-Dirac limit) together with an attractive contact density-density interaction $-|V| \delta\left(\boldsymbol{r}-\boldsymbol{r}^{\prime}\right)$, which induces purely singlet pairing $\Delta_{\mathrm{s}}$ as we will show in Sec. IV A. The gap equation at the chemical potential $\mu$ and the inverse temperature $\beta$ is

$$
1=|V| \nu\left(\omega_{\mathrm{D}}\right) \sum_{\lambda= \pm} \int_{\mu-\omega_{\mathrm{D}}}^{\mu+\omega_{\mathrm{D}}} \frac{d \varepsilon \nu(\varepsilon)}{\nu\left(\omega_{\mathrm{D}}\right)} \frac{\tanh \beta E_{\lambda}(\varepsilon) / 2}{2 E_{\lambda}(\varepsilon)}
$$

where the "Debye" energy cutoff $\omega_{\mathrm{D}}$ has been introduced,

$$
E_{\lambda}(\varepsilon):=\sqrt{(\varepsilon-\lambda \mu)^{2}+\Delta_{\mathrm{S}}^{2}},
$$

and

$$
\nu(\varepsilon):=\frac{|\varepsilon|}{2 \pi\left(\hbar v_{\mathrm{RD}}\right)^{2}}
$$

is the Rashba-Dirac density of states per unit area. The temperature dependence of the static Pauli magnetic susceptibility for an out-of-plane uniform applied magnetic field is then given by $\chi_{33}=2 \chi_{11}=2 \chi_{22}$ with

$$
\begin{aligned}
\chi_{33} \propto & \sum_{\lambda= \pm} \int \frac{d^{2} \boldsymbol{k}}{(2 \pi)^{2}} \frac{E_{k \lambda}^{2}+\xi_{k, \lambda} \xi_{k,-\lambda}+\Delta_{\mathrm{S}}^{2}(T)}{E_{k \lambda}} \\
& \times \frac{1}{\xi_{k, \lambda}^{2}-\xi_{k,-\lambda}^{2}} \tanh \frac{\beta E_{k \lambda}}{2} .
\end{aligned}
$$

We plot the temperature dependence of the self-consistent pair potential $\Delta_{\mathrm{s}}(T)$ and of $\chi_{33}(T)$ in Fig. 2. First, we note that $\chi_{33}(T=0) \neq 0$. This is a direct consequence of the spinorbit coupling. ${ }^{17-21}$ Second, we note that $\chi_{33}(T)$ decreases as a function of temperature beyond the critical temperature, i.e., when $T>T_{\mathrm{c}}$. Although the finite value of $\chi_{33}(T=0)$ is typical of noncentrosymmetric superconductors, ${ }^{17-21} \chi_{33}\left(T>T_{\mathrm{c}}\right)$ only saturates to a value proportional to the density of state at the Fermi level in the regime (1.1). In the regime (1.2) the decrease of $\chi_{33}\left(T>T_{\mathrm{c}}\right)$ can be understood with the help of Eq. (3.5) if $\mu$ is substituted for $T$ to mimic thermal population. Indeed, Eq. (3.5) implies that the normal-state $\chi_{33}(\mu)$ at $T=0$ increases with $\mu$ if the Fermi level is below the Rashba-Dirac point, but decreases with $\mu$ if the Fermi level is above the Rashba-Dirac point, for the states above the Rashba-Dirac point give a contribution that cancels part of the susceptibility coming from the states below the Rashba-Dirac point.

\section{SUPERCONDUCTING INSTABILITIES}

\section{A. Density-density interaction}

To obtain the BdG Hamiltonian (2.5a) self-consistently, we consider first a density-density interaction given by

$$
H_{V}:=\frac{1}{2} \sum_{q} V_{q} \rho_{q} \rho_{-q}, \quad \rho_{q}:=\sum_{k, s=\uparrow, \downarrow} c_{k+q s}^{\dagger} c_{k s},
$$

where $V_{\boldsymbol{q}}$ is an even function of momentum. Normal ordering yields

$$
H_{V}=\frac{1}{2} \sum_{q} V_{q} \sum_{k, \boldsymbol{k}^{\prime}} \sum_{s, s^{\prime}} c_{\boldsymbol{k}+\boldsymbol{q} s^{\prime}}^{\dagger} c_{\boldsymbol{k}^{\prime}-\boldsymbol{q} s^{\prime}}^{\dagger} c_{\boldsymbol{k}^{\prime} s^{\prime}} c_{\boldsymbol{k} s}+\frac{1}{2} \sum_{\boldsymbol{q}} V_{q} \sum_{\boldsymbol{k}} \sum_{s} c_{\boldsymbol{k} s}^{\dagger} c_{\boldsymbol{k} s} .
$$

After renormalization of the chemical potential and restriction of the normal-ordered interaction to the scattering of Cooper pairs with vanishing center-of-mass momentum, we obtain the reduced Hamiltonian

$$
H_{V}^{\mathrm{red}}=\frac{1}{2} \sum_{k, p} V_{k-p} \sum_{s, s^{\prime}} c_{k s}^{\dagger} c_{-k s^{\prime}}^{\dagger} c_{-p s^{\prime}} c_{p s} .
$$

We show in Appendix A that the reduced interaction (4.3) has the representation

$$
\begin{aligned}
H_{V}^{\mathrm{red}}= & \frac{1}{2} \sum_{k, p} \sum_{\lambda, \lambda^{\prime}= \pm 1} V_{k-p} e^{i\left(\varphi_{p}-\varphi_{k}\right)}\left[\cos \left(\varphi_{p}-\varphi_{k}\right)+\lambda \lambda^{\prime}\right] \\
& \times a_{k \lambda}^{\dagger} a_{-k \lambda}^{\dagger} a_{-p \lambda^{\prime}} a_{p \lambda^{\prime}}+\cdots
\end{aligned}
$$

The terms $\cdots$ that were omitted involve pairs of creation or of annihilation operators of opposite helicities. We ignore these terms because we are going to perform a mean-field approximation for Cooper pairs made of time-reversed helicity single-particle states from Eq. (2.7c).

We define the mean-field superconducting order parameters to be

$$
\widetilde{\delta}_{k \lambda}:=\lambda e^{i \varphi_{-k}}\left\langle a_{-k \lambda} a_{k \lambda}\right\rangle_{\beta, \mu}=+\widetilde{\delta}_{-k \lambda},
$$

where $\lambda= \pm$. The angular bracket represents the statistical averaging at inverse temperature $\beta$ and chemical potential $\mu$. We also define the mean-field helicity pairing potentials to be 


$$
\widetilde{\Delta}_{k \lambda}:=\frac{1}{2} \sum_{p, \lambda^{\prime}} V_{k-p}\left[\lambda \lambda^{\prime} \cos \left(\varphi_{p}-\varphi_{k}\right)+1\right] \widetilde{\delta}_{p \lambda^{\prime}}=\widetilde{\Delta}_{-k \lambda}
$$

where $\lambda= \pm$. The mean-field superconducting order parameter $(4.5 \mathrm{a})$ and the pairing potentials $(4.5 \mathrm{~b})$ enter the (meanfield) BdG Hamiltonian of the form (2.12) and obey the selfconsistent conditions

$$
\widetilde{\delta}_{p \lambda}=-\frac{\tilde{\Delta}_{p \lambda}}{2 E_{p \lambda}} \tanh \left(\beta E_{p \lambda} / 2\right)
$$

where the single-particle dispersion in the superconducting state $E_{p \lambda}$ is defined in Eq. (2.13).

If the pairing interaction is independent of momentum (i.e., a contact interaction in space), the summation over $\boldsymbol{p}$ on the right-hand side of Eq. (4.5b) cancels the dependence on $\lambda$. Hence, both order parameters are then equal $\widetilde{\Delta}_{k+}=\widetilde{\Delta}_{k-}$ and we can see from the transformation $(2.10 \mathrm{~b})$ that the pairing potential will be of pure spin-singlet nature. Observe that this result is independent of the noninteracting part of the Hamiltonian, and thus valid for both models (2.1). It was also found in the context of 3D noncentrosymmetric superconductors in Ref. 55.

We have also solved self-consistently the gap equation with the Dirac dispersion $\left(\varepsilon_{k} \equiv 0\right)$ for a pairing interaction that is isotropic in momentum space $V_{q}=V_{|q|}$. When the chemical potential is much larger than the transition temperature $|\mu \beta| \gg 1$, we have found that the triplet component never exceeds the singlet component of the superconducting pairing potential if the pairing interaction $V_{|q|}$ never changes sign as a function of $|\boldsymbol{q}|$. The latter is true for most of the commonly used model interactions, except Cooper pairing mediated by the Friedel oscillations induced by the screening of the Coulomb repulsive interaction, for example. ${ }^{56,57}$

The density-density interaction as considered here might provide a model for the pairing interaction recently discovered at the superconducting interfaces in $\mathrm{LaAlO}_{3} / \mathrm{SrTiO}_{3}$ (Ref. 58) or in some electrolyte $/ \mathrm{SrTiO}_{3}$ (Ref. 59) that feature a low density and high mobility of the charge carriers.

\section{B. Heisenberg interaction}

As a second example, we study the $\mathrm{SU}(2)$ preserving spin-density-spin-density interaction

$$
H_{\mathrm{H}}:=\frac{1}{2} \sum_{\boldsymbol{q}} J_{\boldsymbol{q}} \boldsymbol{S}_{q} \cdot \boldsymbol{S}_{-q}, \quad \boldsymbol{S}_{q}:=\frac{1}{2} \sum_{k ; s, s^{\prime}} c_{k+q s}^{\dagger} \boldsymbol{\sigma}_{s, s^{\prime}} c_{k s^{\prime}},
$$

where $J_{\boldsymbol{q}}$ is an even function of momentum. Proceeding in the same way as in Sec. IV A, we obtain the reduced Hamiltonian for the scattering of Cooper pairs with vanishing center-of-mass momentum

$$
H_{\mathrm{H}}^{\mathrm{red}}=\frac{1}{8} \sum_{\boldsymbol{k}, \boldsymbol{p}} \sum_{s_{1}, s_{2}, s_{3}, s_{4}} J_{\boldsymbol{k}-\boldsymbol{p}} \boldsymbol{\sigma}_{s_{1}, s_{4}} \cdot \boldsymbol{\sigma}_{s_{2}, s_{3}} c_{\boldsymbol{k} s_{1}}^{\dagger} c_{-\boldsymbol{k} s_{2}}^{\dagger} c_{-\boldsymbol{p} s_{3}} c_{\boldsymbol{p} s_{4}}
$$

As is shown in Appendix A the reduced interaction (4.8) has the following representation in the helicity basis

$$
\begin{aligned}
H_{\mathrm{H}}^{\mathrm{red}}= & \frac{1}{16} \sum_{k, p} \sum_{\lambda, \lambda^{\prime}= \pm 1} J_{k-p} e^{i\left(\varphi_{p}-\varphi_{k}\right)}\left[\cos \left(\varphi_{p}-\varphi_{k}\right)-3 \lambda \lambda^{\prime}\right] \\
& \times a_{k \lambda}^{\dagger} a_{-k \lambda}^{\dagger} a_{-p \lambda^{\prime}} a_{p \lambda^{\prime}}+\cdots
\end{aligned}
$$

The terms $\cdots$ that were omitted, just as in Sec. IV A where we studied density-density interactions, involve pairs of creation or of annihilation operators of opposite helicities. We ignore these terms because we are going to perform a meanfield approximation with pairs of time-reversed helicity single-particle states from Eq. (2.7c).

Again, we define the mean-field superconducting order parameters to be

$$
\widetilde{\delta}_{k \lambda}:=\lambda e^{i \varphi_{-k}}\left\langle a_{-k \lambda} a_{k \lambda}\right\rangle_{\beta, \mu}=+\widetilde{\delta}_{-k \lambda},
$$

where $\lambda= \pm$ and the angular bracket represents the statistical averaging at inverse temperature $\beta$ and chemical potential $\mu$. The mean-field helicity pairing potentials is defined to be

$$
\widetilde{\Delta}_{k \lambda}:=\frac{1}{2} \sum_{p, \lambda^{\prime}} J_{k-p}\left[\lambda \lambda^{\prime} \cos \left(\varphi_{p}-\varphi_{k}\right)-3\right] \widetilde{\delta}_{p \lambda^{\prime}}=\widetilde{\Delta}_{-k \lambda},
$$

where $\lambda= \pm$.

Together with the superconducting order parameters (4.10a) they obey the self-consistent conditions (4.6a).

The term proportional to $\lambda$ represents the triplet component of the pairing potential while the constant term in the square bracket gives the singlet component. We have solved self-consistently the gap equation with the Dirac dispersion (i.e., $\varepsilon_{k} \equiv 0$ ) for a pairing interaction that is isotropic in momentum space $J_{q}=J_{|q|}$. As with the case of the densitydensity interaction, we have found under the assumption $|\mu \beta| \gg 1$ that the triplet component never exceeds the singlet component of the superconducting pairing potential if the pairing interaction $J_{|q|}$ never changes sign as a function of $|\boldsymbol{q}|$.

The Heisenberg interaction is attractive (repulsive) in the spin-singlet channel and repulsive (attractive) in the spintriplet channel for $J_{q}>0\left(J_{q}<0\right)$. In centrosymmetric superconductors, this property leads the way toward spinfluctuation mediated spin-triplet superconductivity for $J_{q}$ $<0$. If inversion symmetry is broken, however, spin-singlet and spin-triplet pairing channels are not separated and for the case $J_{q}<0$, the interaction is altogether not attractive. Hence, the Heisenberg interaction will not lead to triplet (dominated) superconductivity in the same fashion as in centrosymmetric superconductors.

\section{Dzyaloshinskii-Moriya interaction}

Finally, we study a spin-density-spin-density interaction of Dzyaloshinskii-Moriya type, which requires the breaking 
of inversion symmetry to be present. Let the coefficient $\boldsymbol{D}_{\boldsymbol{q}}$ be a three vector with vanishing $z$ component for our case. It shares the symmetry of $\boldsymbol{g}_{\boldsymbol{q}}$, in particular, it is odd under $\boldsymbol{q} \rightarrow-\boldsymbol{q}$. The Dzyaloshinskii-Moriya interaction is then

$$
H_{\mathrm{DM}}:=\frac{1}{2} \sum_{q} D_{q} \cdot\left(S_{q} \wedge S_{-q}\right) .
$$

The reduced Hamiltonian for the scattering of Cooper pairs with vanishing center-of-mass momentum reads

$$
\begin{aligned}
H_{\mathrm{DM}}^{\mathrm{red}}= & \frac{1}{8} \sum_{\boldsymbol{k}, \boldsymbol{p}} \sum_{s_{1}, s_{2}, s_{3}, s_{4}} \boldsymbol{D}_{\boldsymbol{k}-\boldsymbol{p}} \cdot\left(\boldsymbol{\sigma}_{s_{1}, s_{4}} \wedge \boldsymbol{\sigma}_{s_{2}, s_{3}}\right) \\
& \times c_{\boldsymbol{k} s_{1}}^{\dagger} c_{-\boldsymbol{k} s_{2}}^{\dagger} c_{-\boldsymbol{p} s_{2}} c_{\boldsymbol{p} s_{4}} .
\end{aligned}
$$

As is shown in Appendix A, the reduced interaction (4.12) has the representation

$$
\begin{aligned}
H_{\mathrm{DM}}^{\mathrm{red}}= & \frac{i}{8} \sum_{\boldsymbol{k}, \boldsymbol{p}} \sum_{\lambda, \lambda^{\prime}= \pm 1} e^{i\left(\varphi_{\left.p^{-}-\varphi_{k}\right)} \boldsymbol{D}_{k-p}\right.} \cdot\left(\lambda \frac{\boldsymbol{g}_{\boldsymbol{p}}}{\left|\boldsymbol{g}_{\boldsymbol{p}}\right|}-\lambda^{\prime} \frac{\boldsymbol{g}_{\boldsymbol{k}}}{\left|\boldsymbol{g}_{\boldsymbol{k}}\right|}\right) \\
& \times a_{\boldsymbol{k} \lambda}^{\dagger} a_{-k \lambda}^{\dagger} a_{-p \lambda^{\prime}} a_{\boldsymbol{p} \lambda^{\prime}}+\cdots .
\end{aligned}
$$

Once again, the terms $\cdots$ that were omitted involve a pair of creation or of annihilation operators of opposite helicities while we keep only pairs made of time-reversed helicity single-particle states from Eq. (2.7c).

The mean-field superconducting order parameters are again defined to be

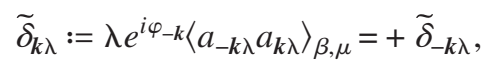

where $\lambda= \pm$ and the angular bracket represents the statistical averaging at inverse temperature $\beta$ and chemical potential $\mu$. We also define the mean-field helicity pairing potentials to be

$$
\widetilde{\Delta}_{k \lambda}:=\frac{1}{2} \sum_{p, \lambda^{\prime}} D_{k-p} \cdot\left(\lambda^{\prime} \frac{g_{p}}{\left|g_{p}\right|}-\lambda \frac{g_{k}}{\left|g_{k}\right|}\right) \widetilde{\delta}_{p \lambda^{\prime}}=\widetilde{\Delta}_{-k \lambda},
$$

where $\lambda= \pm$. Together with the superconducting order parameters (4.14a) they obey the self-consistent conditions (4.6a).

We have solved self-consistently the gap equation with the Dirac dispersion $\left(\varepsilon_{k} \equiv 0\right)$ for the model interaction $\boldsymbol{D}_{q}$ $=D g_{q} \exp \left(-g_{q}^{2} / a^{2}\right)$, where $a$ and $D$ are parameters. We have found that, depending on the chemical potential and the cutoff parameter $a$, the triplet component can exceed the singlet component of the superconducting pairing potential. This is in contrast to the results from the density-density interaction and the Heisenberg interaction, where the singlet component is dominant. However, as the Dzyaloshinskii-Moriya interaction arises in second-order perturbation theory from an exchange interaction, it should not be considered on its own.

\section{Superconductivity with in-plane magnetic field}

A well established result for $2 \mathrm{D}$ noncentrosymmetric superconductors with the Rashba spin-orbit coupling (2.2c) is that the superconducting pair potential acquires a real-space modulation in the presence of a Zeeman coupling to an inplane magnetic field. ${ }^{17,23-25}$ An in-plane magnetic field shifts the Fermi sea away from the center of the Brillouin zone and as a result, the electrons with opposite wave vectors are not degenerate in energy anymore.

A similar effect is expected for the 2D Rashba-Dirac model subject to this study. We shall demonstrate this for a momentum-independent density-density interaction as was discussed in Sec. IV A.

The noninteracting Hamiltonian (2.4a) in the RashbaDirac limit, i.e., for $\varepsilon_{k} \equiv 0$, is altered in the presence of an in-plane magnetic field

$$
\boldsymbol{B} \equiv B_{1} \boldsymbol{e}_{1}+B_{2} \boldsymbol{e}_{2}, \quad \boldsymbol{e}_{3}:=\boldsymbol{e}_{1} \wedge \boldsymbol{e}_{2}, \quad \boldsymbol{g}_{\boldsymbol{k}} \equiv g_{\boldsymbol{k} 1} \boldsymbol{e}_{1}+g_{\boldsymbol{k} 2} \boldsymbol{e}_{2},
$$

according to

$$
\begin{gathered}
H_{0}^{B}=\sum_{k \in \mathrm{BZ}} \psi_{k}^{\dagger} \mathcal{H}_{0 ; k}^{B} \psi_{k}=\sum_{k \in \mathrm{BZ}} \tilde{\psi}_{k}^{\dagger} \tilde{\mathcal{H}}_{0 ; k}^{B} \tilde{\psi}_{k}, \\
\mathcal{H}_{0 ; k}^{B}=-\mu \sigma_{0}+\left(g_{k}-\boldsymbol{B}\right) \cdot \boldsymbol{\sigma} \\
\tilde{\mathcal{H}}_{0 ; k}^{B}=\left(\begin{array}{cc}
\xi_{k+}^{B} & 0 \\
0 & \xi_{k-}^{B}
\end{array}\right) .
\end{gathered}
$$

The single-particle dispersion is now given by

$$
\xi_{k \pm}^{B}=-\mu \pm\left|g_{k}-B\right|
$$

Accordingly, the phase factor entering the transformation, Eq. (2.4c), between the laboratory basis and the helicity basis is changed to

$$
e^{i \varphi_{k}^{B}}=\frac{g_{k 1}-B_{1}+i g_{k 2}-i B_{2}}{\left|g_{k}-B\right|} .
$$

A pair of electrons on the Fermi surface without magnetic field with opposite wave vectors $\boldsymbol{k}$ and $\boldsymbol{- k}$ is not degenerate in energy anymore in the presence of $\boldsymbol{B}$, for

$$
\xi_{k^{ \pm}}^{B}-\xi_{-k_{ \pm}}^{B}=\mp 2 \frac{\boldsymbol{g}_{k} \cdot \boldsymbol{B}}{\left|\boldsymbol{g}_{\boldsymbol{k}}\right|}+\mathcal{O}\left(\frac{|\boldsymbol{B}|^{2}}{\left|\boldsymbol{g}_{k}\right|^{2}}\right) .
$$

It might thus be energetically more favorable to pair electrons with the same energy but with a finite center-of-mass momentum, than to pair electrons with vanishing center-ofmass momentum. For simplicity, we also assume that only electrons of a single helicity $\lambda$ form Cooper pairs. From here on, we denote the center-of-mass momentum of Cooper pairs by $\boldsymbol{q}$ while $\boldsymbol{k}$ and $\boldsymbol{k}^{\prime}$ refer to the relative coordinate of the paired electrons. We assume that a single wave vector $\boldsymbol{q}$ for the modulation of the pairing potential will be energetically favorable, rather than a set of degenerate wave vectors. With these simplifications, the self-consistent gap equation for the pair potential $\widetilde{\Delta}_{k \lambda}(\boldsymbol{q})$ at temperature $T$ close to the superconducting transition temperature and for $N$ sites is 


$$
\begin{aligned}
\widetilde{\Delta}_{k, \boldsymbol{q} ; \lambda}= & -\frac{V}{2} \frac{T}{N} \sum_{\boldsymbol{k}^{\prime}, \omega_{n}} \cos \left(\frac{\varphi_{-\boldsymbol{k}^{\prime}+\boldsymbol{q} / 2}^{\boldsymbol{B}}-\varphi_{-\boldsymbol{k}+\boldsymbol{q} / 2}^{\boldsymbol{B}}}{2}\right) \\
& \times \cos \left(\frac{\varphi_{\boldsymbol{k}^{\prime}+\boldsymbol{q} / 2}^{B}-\varphi_{\boldsymbol{k}+\boldsymbol{q} / 2}^{B}}{2}\right) \widetilde{\Delta}_{\boldsymbol{k}^{\prime}, \boldsymbol{q} ; \lambda} \\
& \times G_{\boldsymbol{k}^{\prime}+\boldsymbol{q} / 2, i \omega_{n} ; \lambda}^{(0)} G_{-\boldsymbol{k}^{\prime}+\boldsymbol{q} / 2,-i \omega_{n} ; \lambda}^{(0)},
\end{aligned}
$$

where the single-particle Green's function in the normal state for electrons with helicity $\lambda= \pm$ is given by

$$
G_{k, i \omega_{n} ; \lambda}^{(0)}=-\frac{1}{-i \omega_{n}+\xi_{k \lambda}^{B}}
$$

For $s$-wave pairing, the pairing potential $\widetilde{\Delta}_{k \lambda}(\boldsymbol{q})$ is independent of $\boldsymbol{k}$. The gap Eq. (4.21) simplifies to, after performing the summation over Matsubara frequencies,

$$
\begin{aligned}
1= & -\frac{V}{2 N} \sum_{\boldsymbol{k}^{\prime}} \cos \left(\frac{\varphi_{-\boldsymbol{k}^{\prime}+\boldsymbol{q} / 2}^{\boldsymbol{B}}-\varphi_{-\boldsymbol{k}+\boldsymbol{q} / 2}^{\boldsymbol{B}}}{2}\right) \\
& \times \cos \left(\frac{\varphi_{\boldsymbol{k}^{\prime}+\boldsymbol{q} / 2}^{B}-\varphi_{k+\boldsymbol{q} / 2}^{B}}{2}\right) f_{\boldsymbol{k}^{\prime}, \boldsymbol{q} ; \lambda}^{\boldsymbol{B}}
\end{aligned}
$$

with the function

$$
f_{\boldsymbol{k}, \boldsymbol{q} ; \lambda}^{\boldsymbol{B}}:=\frac{\tanh \frac{\xi_{-\boldsymbol{k}+(\boldsymbol{q} / 2) \lambda}^{\boldsymbol{B}}}{2 T}+\tanh \frac{\xi_{k+(\boldsymbol{q} / 2) \lambda}^{\boldsymbol{B}}}{2 T}}{2\left(\xi_{-\boldsymbol{k}+(\boldsymbol{q} / 2) \lambda}^{\boldsymbol{B}}+\xi_{\boldsymbol{k}+(\boldsymbol{q} / 2) \lambda}^{\boldsymbol{B}}\right)} .
$$

In the Rashba-Dirac continuum limit (2.1) the dispersion (4.17) together with the transformation (4.18) and the thermal factor $(4.22 \mathrm{~b})$ obey the symmetries

$$
\begin{gathered}
\xi_{k+\left(\boldsymbol{q}_{0} / 2\right) \lambda}^{\boldsymbol{B}}=\xi_{-\boldsymbol{k}+\left(\boldsymbol{q}_{0} / 2\right) \lambda}^{\boldsymbol{B}}=\xi_{k \lambda}^{\boldsymbol{B}=0}=\xi_{-k \lambda}^{\boldsymbol{B}=0}, \\
\varphi_{\boldsymbol{k}+\left(\boldsymbol{q}_{0} / 2\right)}^{\boldsymbol{B}}=\varphi_{\boldsymbol{k}}^{\boldsymbol{B}=0}, \\
f_{\boldsymbol{k},\left(\boldsymbol{q}_{0} / 2\right) ; \lambda}^{\boldsymbol{B}}=f_{\boldsymbol{k}, 0 ; \lambda}^{\boldsymbol{B}=0},
\end{gathered}
$$

with $\boldsymbol{q}_{0}=2 \boldsymbol{B} \wedge \boldsymbol{e}_{3} /\left(\hbar v_{\mathrm{RD}}\right)$ being proportional to the shift of the Fermi surface induced by $\boldsymbol{B}$. Hence, the gap equation for the superconducting condensate with the center-of-mass momentum $\boldsymbol{q}_{0}$ in the presence of the in-plane magnetic field $\boldsymbol{B}$ is the same as the gap equation in the absence of any in-plane magnetic field for a condensate with vanishing center-ofmass momentum. A condensate with vanishing center-ofmass momentum has the largest transition temperature. Hence, we deduce from the symmetry (4.23) that a superconducting order parameter with the center-of-mass momentum $\boldsymbol{q}_{0}$ nucleates in the presence of an in-plane magnetic field. The wave vector of the modulated pairing potential is perpendicular to the magnetic field in the plane and is independent of the chemical potential. It also follows that the critical temperature of superconductivity is not suppressed by the magnetic field in the Rashba-Dirac continuum limit (2.1) and by the consideration of only one helicity.

In contrast to this simple result, the center-of-mass momentum of Cooper pairs in 2D noncentrosymmetric super- conductors with the Rashba spin-orbit coupling (2.2c) is selected by a delicate energetical compromise on how the two helicity-resolved Fermi surfaces are shifted in opposite directions in momentum space.

\section{MEAN-FIELD PHASE DIAGRAM}

It is time to explore the mean-field phase diagram that follows from Hamiltonian (2.10a) in the parameter space spanned by the choice made for the normal-state dispersion and for the pair potentials. To this end, we consider the parameter space spanned by the couplings $\mu, \Delta_{\mathrm{s}}$, and $\Delta_{\mathrm{t}}$ entering the mean-field Hamiltonian. A mean-field phase corresponds to a connected region in parameter space characterized by a nonvanishing gap. We shall then introduce in Sec. VI point defects in the mean-field Hamiltonian (2.10a), i.e., superconducting vortices with unit circulation, and compute the parity of the number of zero modes they bind to characterize the topological nature of the mean-field phases separated in parameter space by gap-closing boundaries.

For convenience, we recall that the BdG Hamiltonian is, in the $\Phi$ representation, Eq. (2.10a),

$$
\mathcal{H}_{k}:=\left(\begin{array}{cccc}
\varepsilon_{k}-\mu & A_{k} e^{-i \varphi_{k}} & \Delta_{\mathrm{s}, k} & \Delta_{\mathrm{t}, k} e^{-i \varphi_{k}} \\
A_{k} e^{i \varphi_{k}} & \varepsilon_{k}-\mu & \Delta_{\mathrm{t}, k} e^{+i \varphi_{k}} & \Delta_{\mathrm{s}, \boldsymbol{k}} \\
\Delta_{\mathrm{s}, \boldsymbol{k}} & \Delta_{\mathrm{t}, \boldsymbol{k}} e^{-i \varphi_{k}} & -\varepsilon_{k}+\mu & -A_{k} e^{-i \varphi_{k}} \\
\Delta_{\mathrm{t}, \boldsymbol{k}} e^{+i \varphi_{k}} & \Delta_{\mathrm{s}, \boldsymbol{k}} & -A_{\boldsymbol{k}} e^{+i \varphi_{k}} & -\varepsilon_{k}+\mu
\end{array}\right),
$$

where the normal-state dispersion is specified by

$$
\begin{gathered}
\varepsilon_{k}=\varepsilon_{-k} \in \mathbb{R}, \quad \mu \in \mathbb{R}, \\
A_{k} \equiv\left|g_{k}\right|=A_{-k} \geq 0, \quad g_{k}=-g_{-k} \in \mathbb{R}^{2}, \\
\varphi_{k} \equiv \arctan \frac{g_{k ; 2}}{g_{k ; 1}} \in[0,2 \pi[,
\end{gathered}
$$

the singlet-pair potential $\Delta_{\mathrm{s}, \boldsymbol{k}}$ and the triplet-pair potential $\Delta_{\mathrm{t}, \boldsymbol{k}}$ transform according to any trivial irreducible representation of the space group consistent with $\Delta_{\mathrm{t}, k} e^{ \pm i \varphi_{k}}$ being single valued. In the isotropic continuum limit, we thus assume that the singlet-pair potential is constant while the triplet-pair potential $\Delta_{\mathrm{t}}(\boldsymbol{k})$ can be factorized into a real number $\Delta_{\mathrm{t}}$ times some strictly increasing positive function $f$ with at least a first-order zero at the origin such that (i) it saturates to unity for large positive argument and (ii) is invertible on the positive real axis with the inverse $f^{-1}$, say, for instance, $f(x):=\tanh x$, i.e.,

$$
\Delta_{\mathrm{t}}(\boldsymbol{k})=\Delta_{\mathrm{t}} f\left(|\boldsymbol{k}| / k_{\mathrm{t}}\right)
$$

for some wavelength $k_{\mathrm{t}}>0$ that defines the size of the core of the vortex $\exp [-i \varphi(k)]$ at the origin in $\boldsymbol{k}$ space. The aim of this section is to identify when the quasiparticle spectral gap vanishes as a function of the parameters $\Delta_{\mathrm{s}}, \Delta_{\mathrm{t}}$, and $\mu$ for a given dispersion relation in the isotropic continuum limit, i.e., we need the eigenvalues of Eq. (5.1).

To this end, we first square both sides of Eq. (5.1), finding the block diagonal form 


$$
\begin{gathered}
\mathcal{H}_{k}^{2}=\left(\begin{array}{cc}
\mathrm{A}_{\boldsymbol{k}} & 0 \\
0 & \mathrm{~A}_{\boldsymbol{k}}
\end{array}\right), \\
\mathrm{A}_{\boldsymbol{k}}=\left[\left(\varepsilon_{\boldsymbol{k}}-\mu\right)^{2}+A_{k}^{2}+\Delta_{\mathrm{s}}^{2}+\Delta_{\mathrm{t}, k}^{2}\right] \sigma_{0}+2\left[\left(\varepsilon_{k}-\mu\right) A_{k}+\Delta_{\mathrm{s}} \Delta_{\mathrm{t}, \boldsymbol{k}}\right] \\
\times \cos \varphi_{k} \sigma_{1}+2\left[\left(\varepsilon_{k}-\mu\right) A_{k}+\Delta_{\mathrm{s}} \Delta_{\mathrm{t}, k}\right] \sin \varphi_{k} \sigma_{2} .
\end{gathered}
$$

The four eigenvalues of $\mathcal{H}_{\boldsymbol{k}}$ are

$$
E_{k ; \lambda, \pm}= \pm \sqrt{\left(\varepsilon_{k}-\mu+\lambda A_{k}\right)^{2}+\left(\Delta_{\mathrm{s}}+\lambda \Delta_{\mathrm{t}, k}\right)^{2}},
$$

where $\lambda= \pm$. All nonvanishing energy eigenvalues come in pairs with opposite signs. This spectral symmetry is a consequence of the particle-hole transformation $\left[X_{\mu \nu}\right.$ was defined in Eq. (3.8b)]

$$
X_{22} \mathcal{H}_{-k}^{T} X_{22}=-\mathcal{H}_{k} .
$$

The Hamiltonian $\mathcal{H}_{k}$ also features a helical symmetry given by

$$
\left(\hat{g}_{k 1} X_{10}+\hat{g}_{k 2} X_{20}\right) \mathcal{H}_{k}\left(\hat{g}_{k 1} X_{10}+\hat{g}_{k 2} X_{20}\right)=\mathcal{H}_{k} .
$$

Viewing the Rashba spin-orbit coupling as a fictitious magnetic field along a $\boldsymbol{k}$-dependent direction, the helical symmetry (5.5) reflects the conservation of spin along this direction in momentum space.

For completeness, TRS is nothing but

$$
X_{20} \mathcal{H}_{-k}^{*} X_{20}=+\mathcal{H}_{k}
$$

in the $\Phi$ basis of Eq. (2.5b) that we have chosen.

Zero modes are vanishing eigenvalues of $\mathcal{H}_{k}$, i.e., they are the solutions to

$$
\begin{aligned}
0=\operatorname{det} \mathcal{H}_{k}= & {\left[\left(\varepsilon_{k}-\mu+A_{k}\right)^{2}+\left(\Delta_{\mathrm{s}}+\Delta_{\mathrm{t}, k}\right)^{2}\right] } \\
& \times\left[\left(\varepsilon_{k}-\mu-A_{k}\right)^{2}+\left(\Delta_{\mathrm{s}}-\Delta_{\mathrm{t}, k}\right)^{2}\right] .
\end{aligned}
$$

There are thus two possibilities to get zero modes. Either

$$
\operatorname{case}(+): \quad 0=\varepsilon_{k}-\mu+A_{k}, \quad 0=\Delta_{\mathrm{s}}+\Delta_{\mathrm{t}, k},
$$

or

$$
\operatorname{case}(-): \quad 0=\varepsilon_{k}-\mu-A_{k}, \quad 0=\Delta_{\mathrm{s}}-\Delta_{\mathrm{t}, k} .
$$

Equation (5.8a) requires that the $\lambda=+$ helicity gap vanishes on the $\lambda=+$ helicity Fermi surface. Equation $(5.8 \mathrm{~b})$ requires that the $\lambda=-$ helicity gap vanishes on the $\lambda=-$ helicity Fermi surface. The condition

$$
\varepsilon_{k}-\mu+\lambda A_{k}=0
$$

on the normal-state dispersion determines the Fermi surfaces

$$
\mathrm{FS}_{\lambda}:=\left\{\boldsymbol{k} \mid \varepsilon_{k}-\mu+\lambda A_{k}=0\right\} .
$$

The condition

$$
\Delta_{\mathrm{s}}+\lambda \Delta_{\mathrm{t}} f_{k}=0
$$

on the pairing potentials determines the momenta for which the superconducting single-particle gap vanishes

$$
\mathrm{SC}_{\lambda}:=\left\{\boldsymbol{k} \mid \Delta_{\mathrm{s}} \pm \Delta_{\mathrm{t}} f_{k}=0\right\} .
$$

Conditions $(+)$ or $(-)$ are satisfied along the points

$$
\mathrm{FS}_{\lambda} \cap \mathrm{SC}_{\lambda} \neq \varnothing, \quad \lambda= \pm
$$

(in other words, the Fermi surfaces cross the superconducting single-particle nodal surfaces).

\section{A. Isotropic continuum limit}

We work in the continuum limit with the upper bound $\Lambda$ and the lower bound $-\Lambda$ to the single-particle meanfield spectrum, as is appropriate for the surface states of a 3D band insulator. We assume that the SRS dispersion $\varepsilon$, the Rashba dispersion $A$, and profile $f$ of the vortex $\exp [-i \varphi(\boldsymbol{k})]$ at the origin in $\boldsymbol{k}$ space are smooth functions of $|\boldsymbol{k}|$. For the analysis to come, it is useful to define the dimensionless quantity

$$
\mathrm{k} \equiv|\boldsymbol{k}| / k_{\mathrm{t}}
$$

We define the 2D parameter space with $\Delta_{\mathrm{t}} / \Delta_{\mathrm{s}}$ as the horizontal axis and $\mu$ as the vertical axis. For any finite positive singlet pairing potential $\Delta_{\mathrm{s}} \neq 0$, we show

(1) That there are two nonintersecting curves (to simplify the notation $\Lambda \rightarrow \infty$ )

$$
\begin{gathered}
\mu_{+}:(-\infty,-1] \rightarrow \mathbb{R}, \quad \Delta_{\mathrm{t}} / \Delta_{\mathrm{s}} \mapsto \mu_{+}\left(\Delta_{\mathrm{t}} / \Delta_{\mathrm{s}}\right), \\
\mu_{-}:[1, \infty) \rightarrow \mathbb{R}, \quad \Delta_{\mathrm{t}} / \Delta_{\mathrm{s}} \mapsto \mu_{-}\left(\Delta_{\mathrm{t}} / \Delta_{\mathrm{s}}\right),
\end{gathered}
$$

defined by the condition (5.8a) for $\lambda=+$ and (5.8b) for $\lambda=-$ at which the BdG spectrum (5.3) is gapless.

(2) The curves $\mu_{\lambda}$ are one-to-one reparameterizations of the dispersions $\xi_{\lambda}(|\boldsymbol{k}|)$ with $\lambda= \pm$.

(3) How the two curves $\mu_{\lambda}$ change upon changing the topology of the Fermi surfaces.

For the superconducting single-particle gap to vanish, we must choose

$$
\lambda=-\operatorname{sgn} \frac{\Delta_{\mathrm{s}}}{\Delta_{\mathrm{t}}}
$$

in Eq. (5.10a) from which the implicit definition

$$
0 \leq f(\mathrm{k})=\left|\frac{\Delta_{\mathrm{s}}}{\Delta_{\mathrm{t}}}\right|
$$

of $\mathrm{k}$ follows. Hence, $\mathrm{k}$ is the function

$$
\mathrm{k}:(1, \infty) \rightarrow \mathbb{R}, \quad\left|\frac{\Delta_{\mathrm{t}}}{\Delta_{\mathrm{s}}}\right| \rightarrow \mathrm{k}\left(\left|\frac{\Delta_{\mathrm{t}}}{\Delta_{\mathrm{s}}}\right|\right):=f^{-1}\left(\left|\frac{\Delta_{\mathrm{s}}}{\Delta_{\mathrm{t}}}\right|\right),
$$

which is not defined whenever the superconducting singleparticle gap does not close, i.e., when $\left|\Delta_{\mathrm{t}}\right|<\left|\Delta_{\mathrm{S}}\right|$. Claims (1) and (2) follow with the definition

$$
\mu_{\lambda}(\mathrm{k}):=\varepsilon(\mathrm{k})+\lambda A(\mathrm{k}),
$$

where $\mathrm{k}$ and $\lambda$ were themselves defined in Eq. (5.14) and with the momentum core size $k_{\mathrm{t}}$ taken to be unity.

To illustrate how the topology of the normal-state dispersion changes the curves $\mu_{\lambda}$ with $\lambda= \pm$, we make the (electronlike) parabolic approximation 


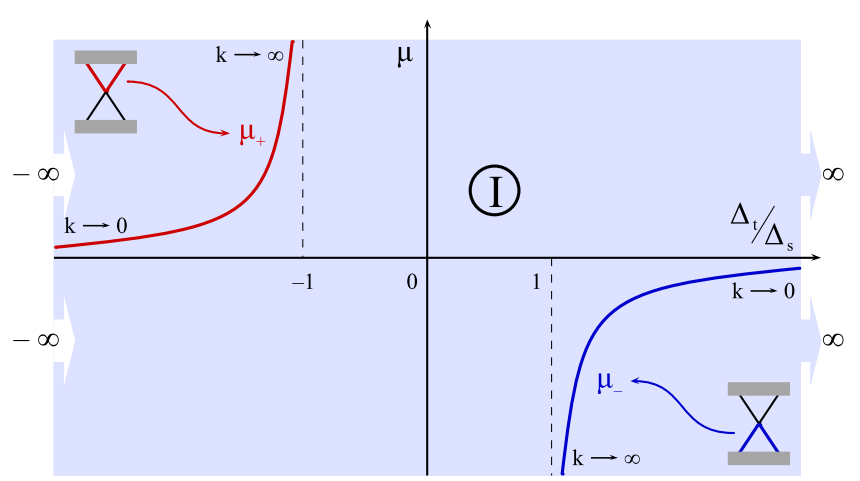

FIG. 3. (Color online) Mean-field phase boundary in the Rashba-Dirac limit, Eq. (5.19).

$$
\varepsilon(\boldsymbol{k})=\frac{\hbar^{2}|\boldsymbol{k}|^{2}}{2 m}, \quad m \geq 0, \quad A(\boldsymbol{k})=\hbar v_{\mathrm{RD}}|\boldsymbol{k}|,
$$

and choose the momentum-vortex profile

$$
f(x)=\tanh x
$$

with the momentum core size $k_{\mathrm{t}}$ taken to be unity. We consider the Rashba-Dirac limit

$$
m=\infty
$$

first, as is illustrated in Fig. 3. The two curves $\mu_{ \pm}\left(\Delta_{t} / \Delta_{\mathrm{s}}\right)$ where the gap vanishes are indicated in Fig. 3. They are obtained via the reparameterization of the dispersions $\xi_{ \pm}(|\boldsymbol{k}|)$, as depicted on the insets on the second and fourth quadrants. There is a one-to-one correspondence between the thick lines in the insets and the curves $\mu_{ \pm}\left(\Delta_{\mathrm{t}} / \Delta_{\mathrm{s}}\right)$.

In Fig. 3, we look at the mean-field phases that can be identified given the gap-closing curves $\mu_{ \pm}\left(\Delta_{\mathrm{t}} / \Delta_{\mathrm{s}}\right)$. Here, one must notice that taking $\Delta_{\mathrm{t}} / \Delta_{\mathrm{s}} \rightarrow \infty$, for a given chemical potential $\mu$ such that the gap does not close, is connected to the path originating from $\Delta_{\mathrm{t}} / \Delta_{\mathrm{s}} \rightarrow-\infty$. For instance, one can send $\Delta_{\mathrm{s}} \rightarrow 0$ so that it changes sign while holding $\Delta_{\mathrm{t}}$ constant but with a given $\mu$ such that the gap does not close. Therefore, the regions depicted in Fig. 3 are connected upon folding the horizontal axis into a circle (the plane into a cylinder). Then, because of the topology of the curves $\mu_{ \pm}\left(\Delta_{t} / \Delta_{\mathrm{s}}\right)$, any one region can be connected to any other without crossing these curves, and hence there is a single phase for the system, which we denote by I.

For any finite curvature of the dispersion $\varepsilon(\boldsymbol{k})$, i.e.,

$$
0 \leq m<\infty,
$$

we find the boundaries shown in Fig. 4. Again, the two curves $\mu_{ \pm}\left(\Delta_{\mathrm{t}} / \Delta_{\mathrm{s}}\right)$ where the gap vanishes are obtained via the reparameterization of the dispersions $\xi_{ \pm}(|\boldsymbol{k}|)$, as depicted on the insets on the second and fourth quadrants. We see that the topology of the curve $\mu_{+}\left(\left|\Delta_{t} / \Delta_{s}\right|\right)$ that tracks the normalstate dispersion $\xi_{+}(|\boldsymbol{k}|)$ is insensitive to tuning $m$ from infinity to any finite value. This is not so for the topology of the curve $\mu_{-}\left(\left|\Delta_{t} / \Delta_{s}\right|\right)$ that tracks the normal-state dispersion $\xi_{-}(|\boldsymbol{k}|)$. This curve is dramatically influenced by the nonmonotonous dependence of $\xi_{-}(|\boldsymbol{k}|)$ on $|\boldsymbol{k}|$ for any finite curvature, i.e., any mass $m<\infty$. In the Rashba-Dirac limit $m=\infty$,

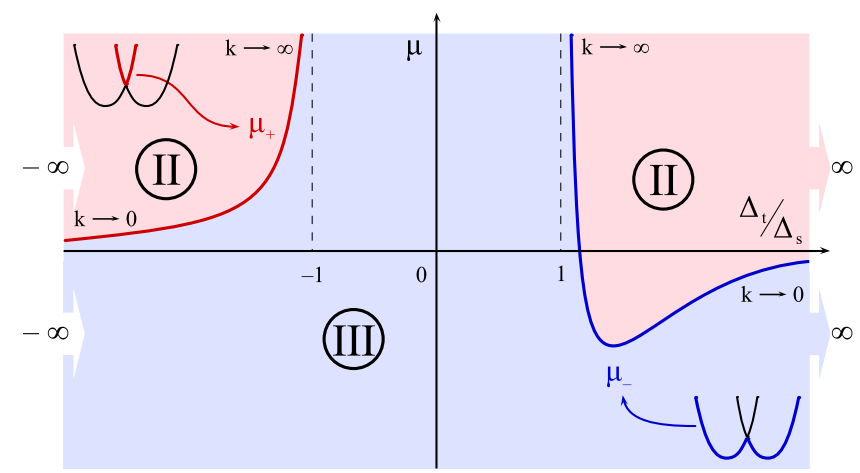

FIG. 4. (Color online) Mean-field phase boundary away from the Rashba-Dirac limit, i.e., when Eq. (5.20) holds.

$\mu_{-}\left(\left|\Delta_{\mathrm{t}} / \Delta_{\mathrm{S}}\right|\right)$ is strictly negative, and $\mu_{-}\left(1_{+}\right) \rightarrow-\infty$. But when $m$ is finite, $\mu_{-}\left(1_{+}\right) \rightarrow \infty$.

The distinct phases in the $m$ finite case are identified in Fig. 4. If the regions with $\Delta_{\mathrm{t}} / \Delta_{\mathrm{s}} \rightarrow \pm \infty$ are identified, then the two regions II and III are always separated from each other by the two curves $\mu_{ \pm}\left(\left|\Delta_{t} / \Delta_{\mathrm{s}}\right|\right)$. There is no path connecting the two regions II and III without ever closing the mean-field spectral gap: this is a necessary (but not sufficient) condition for these to be two distinct phases.

\section{B. Anisotropic case}

The boundaries in the $\Delta_{\mathrm{t}}-\mu$ plane at which the $\mathrm{BdG}$ single-particle spectrum closes for an anisotropic continuum dispersion or for a two-dimensional lattice are more difficult to determine. Indeed, a technical difficulty brought about by the loss of continuous rotational symmetry is that it is not possible anymore to characterize the nodes of the normalstate dispersion or the nodes of the superconducting gaps with a single wave number. This could result in these boundaries acquiring a thickness (i.e., a finite area). ${ }^{60}$

For a 2D lattice model, a qualitative difference with the continuum limit that is of no relevance to this section is the fermion doubling and its consequences for the existence and the stability of Majorana fermions in the core of superconducting vortices. This is the subject of the ensuing section in which we search for Majorana modes in the core of defects (vortices) of the superconducting order parameter and we probe their stability under adiabatic changes of the bulk parameters (i.e., far away from the vortices).

\section{MAJORANA FERMIONS}

Caroli et al. showed in Ref. 37 that isolated vortices in a weakly coupled type II $s$-wave superconductor with TRS and SRS support a discrete set of finite-energy bound states with a level spacing of order of the ratio of the squared singleparticle bulk superconducting gap to the bandwidth. There is no bound state at the Fermi energy bound to the core of vortices in this case.

Jackiw and Rossi showed in Ref. 38 that, in two space and one time dimensions quantum electrodynamics $\left(\mathrm{QED}_{2+1}\right)$ coupled to one scalar Higgs field, an isolated static defect in the Higgs field, i.e., a single vortex with vorticity $N$, 
supports $N$ bound states that are all pinned to the zero energy. These $N$ bound states are $N$ Majorana fermions. An index theorem for this result was proved by Weinberg. ${ }^{39}$

Read and Green ${ }^{42}$ showed that a two-dimensional chiral $p_{x} \pm i p_{y}$ superconductor supports a Majorana mode bound to the core of an isolated half vortex. We are going to show that (i) an isolated vortex with unit vorticity in the singlet-pair potential binds a single Majorana mode in region I of Fig. 3, (ii) an isolated vortex with unit vorticity in the triplet-pair potential binds two Majorana modes in region II of Fig. 4, and (iii) isolated vortices in region III of Fig. 4 do not bind Majorana fermions. We will start by reviewing the derivation of the Jackiw-Rossi Majorana mode that applies to region I of Fig. 3. We will then discuss region II and III in Fig. 4.

We work in the isotropic continuum limit with the Hamiltonian in the $\Phi$ representation (2.10a) given by

$$
\mathcal{H}_{\mathrm{vor}}:=\left(\begin{array}{cccc}
\varepsilon(k, \bar{k}) & \hbar v_{\mathrm{RD}} \bar{k} & \Delta_{\mathrm{s}}(z, \bar{z}) & \frac{1}{2}\left\{\Delta_{\mathrm{t}}(z, \bar{z}), f\left(\frac{|k|}{k_{\mathrm{t}}}\right) \frac{\bar{k}}{|k|}\right\} \\
\hbar v_{\mathrm{RD}} k & \varepsilon(k, \bar{k}) & \frac{1}{2}\left\{\Delta_{\mathrm{t}}(z, \bar{z}), f\left(\frac{|k|}{k_{\mathrm{t}}}\right) \frac{k}{|k|}\right\} & \Delta_{\mathrm{s}}(z, \bar{z}) \\
\text { H.c. } & \text { H.c. } & -\varepsilon(k, \bar{k}) & -\hbar v_{\mathrm{RD}} \bar{k} \\
\text { H.c. } & \text { H.c. } & -\hbar v_{\mathrm{RD}} k & -\varepsilon(k, \bar{k})
\end{array}\right) .
$$

Here, the SRS normal-state dispersion is parabolic

$$
\varepsilon(k, \bar{k}):=\frac{\hbar^{2}|k|^{2}}{2 m}-\mu,
$$

where the real valued $\mu$ is the chemical potential. Moreover, the singlet-pair potential $\Delta_{\mathrm{s}}(z, \bar{z})$ has a unit vortex at the origin of the complex- $z$ plane with the characteristic core size $\ell_{\mathrm{s}}$ and saturates to the bulk value $\Delta_{\mathrm{s}}$ for $|z| \gg \ell_{\mathrm{s}}$, as does the triplet-pair potential $\Delta_{\mathrm{t}}(z, \bar{z})$ with the characteristic core size $\ell_{\mathrm{t}}$ and the bulk value $\Delta_{\mathrm{t}}$ for $|z| \gg \ell_{\mathrm{t}}$. The bulk values $\Delta_{\mathrm{s}}$ and $\Delta_{\mathrm{t}}$ share a common phase that can be removed by a global gauge transformation up to a relative sign. The function $f$ that guarantees single valuedness of the Hamiltonian was defined in Eq. (5.1c). The anticommutators in the antidiagonal are needed since translation invariance has been broken. We are using the complex notation

$$
\begin{aligned}
& k:=k_{1}+i k_{2}, \quad \bar{k}:=k_{1}-i k_{2}, \\
& z:=z_{1}+i z_{2}, \quad \bar{z}:=z_{1}-i z_{2},
\end{aligned}
$$

together with the algebra

$$
\left[z_{a}, k_{b}\right]=i \delta_{a b}, \quad a, b=1,2,
$$

or, equivalently,

$$
[z, \bar{k}]=[\bar{z}, k]=2 i, \quad[z, k]=[\bar{z}, \bar{k}]=0 .
$$

A representation of the algebra (6.2c) is given by

$$
k=-2 i \partial_{\bar{z}}, \quad \bar{k}=-2 i \partial_{z} .
$$

The representation dual to Eq. (6.2d) is

$$
z=2 i \partial_{k}^{-}, \quad \bar{z}=2 i \partial_{k} .
$$

We shall rely on the polar coordinate representation

$$
k=\kappa e^{+i \varphi}
$$

in terms of which

$$
\begin{aligned}
& z=2 i \partial_{k}^{-}=i e^{+i \varphi}\left(\partial_{\kappa}+\frac{i}{\kappa} \partial_{\varphi}\right), \\
& \bar{z}=2 i \partial_{k}=i e^{-i \varphi}\left(\partial_{\kappa}-\frac{i}{\kappa} \partial_{\varphi}\right) .
\end{aligned}
$$

We choose to represent $z, \bar{z}$ as differential operators of functions of $k, \bar{k}$ instead of the other way around because this can bring a simplification in the solution of the zero modes. By solving for the wave functions of the zero modes in momentum space, we take advantage of the fact that we have a first-order differential equation instead of a second-order one, which would be the case had we chosen to solve for the wave functions in position space. This simplification works because we deform the profile of the vortex without changing the fact that the solutions are precisely at energy $E=0$, as we discuss below.

Instead of facing the difficulty to solve analytically for the spectrum of the BdG Hamiltonian (6.1), we are thus going to make approximations that are motivated by the mean-field phase diagram of Sec. V.

We are first going to take the Rashba-Dirac limit at $\mu$ $=0$ (the Rashba-Dirac point) without triplet-pair potential, $\Delta_{\mathrm{t}}=0$. This is nothing but the origin of region I in Fig. 3. In this limit, Hamiltonian (6.1) is the direct sum of two $2 \times 2$ Hamiltonians.

We are then going to take the Fermi limit $v_{\mathrm{RD}}=0$ with $\mu>0$ without singlet-pair potential, $\Delta_{\mathrm{s}}=0$, i.e., the vertical half line at infinity in region I of Fig. 3. In this limit, Hamiltonian (6.1) is again the direct sum of two $2 \times 2$ Hamiltonians. 


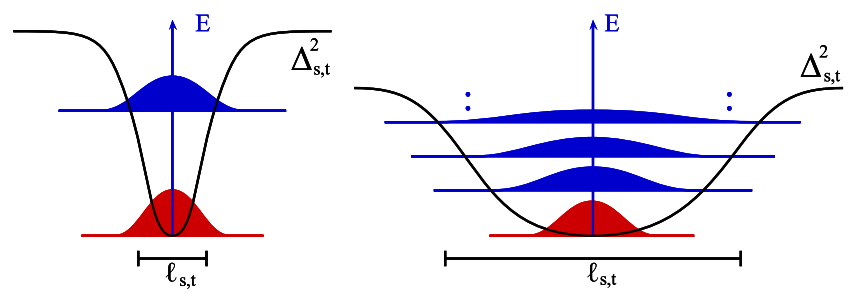

FIG. 5. (Color online) Qualitative comparison of the bound-state spectra of a deep and narrow vortex (left) and a wide and shallow vortex (right). While the former supports only few finite-energy bound states (blue), in the spectrum of the latter more states are allowed. However, the existence of a zero-energy mode (red) is independent of the details of the regime.

Even after these simplifications, the spectrum with a vortex at the origin is difficult to compute. One must solve a system of two coupled partial differential equations that depends on the nonuniversal details encoded by the profile of the vortices in the superconducting pair potentials and by the profile $f$ for the vortex in the $\boldsymbol{d}$ vector. This microscopic information does influence the discrete spectrum that represents the bound states with nonvanishing energies attached to the core of the vortex. For example, if the profile of the vortex is deep due to a large bulk gap and narrow due to a small characteristic size, there should be very few bound states with nonvanishing energies (see Fig. 5). On the other hand, in the opposite limit of a shallow and smooth profile for the vortex, many bound states with nonvanishing energies extending far away from the vortex core are to be expected (see Fig. 5).

However, we are not after the full spectrum of states bound to the core of a vortex. We are only seeking the conditions under which Majorana bound states, i.e., bound states pinned at the normal-state chemical potential, are present. The very existence of a Majorana state does not depend on the profiles of the vortices in real and momentum space as long as the nonvanishing energy spectrum of bound states remains discrete and separated from the zero energy. This suggests choosing the vortex profile

$$
\Delta_{\mathrm{s}, \mathrm{t}}(z, \bar{z})=\Delta_{\mathrm{s}, \mathrm{t}} \frac{z}{\ell_{\mathrm{s}, \mathrm{t}}}
$$

for the singlet $(\mathrm{s})$ or triplet $(\mathrm{t})$ component of the pair potential in real space, respectively, and the vortex profile

$$
f\left(\frac{|k|}{k_{\mathrm{t}}}\right)=\frac{|k|}{k_{\mathrm{t}}}
$$

for the $\boldsymbol{d}$ vector in momentum space. This approximation has the merit of linearizing the spectral eigenvalue problem.

\section{A. Rashba-Dirac limit}

The Rashba-Dirac limit is defined by the condition

$$
m=\infty .
$$

The Rashba-Dirac-point limit is defined by the additional condition

$$
\mu=0 .
$$

In this limit and when the singlet-pair potential vanishes, the bulk gap closes so that vortices in the triplet-pair potential are ill defined.

In the limit (6.6) and when the triplet-pair potential vanishes, after setting $\hbar=v_{\mathrm{RD}}=1$,

$$
\mathcal{H}_{\text {vor }}:=\left(\begin{array}{cccc}
0 & \bar{k} & \frac{\Delta_{\mathrm{s}}}{\ell_{\mathrm{s}}} z & 0 \\
k & 0 & 0 & \frac{\Delta_{\mathrm{s}}}{\ell_{\mathrm{s}}} \\
\text { H.c. } & 0 & 0 & -\bar{k} \\
0 & \text { H.c. } & -k & 0
\end{array}\right)
$$

decomposes into the direct sum of the $2 \times 2$ Hermitian operators

$$
\mathcal{H}_{\mathrm{vor}}^{(1)}:=\left(\begin{array}{cc}
\bar{k} & \frac{\Delta_{\mathrm{s}}}{\ell_{\mathrm{s}}} z \\
\frac{\bar{\Delta}_{\mathrm{s}}}{\ell_{\mathrm{s}}} & -k
\end{array}\right)
$$

and

$$
\mathcal{H}_{\mathrm{vor}}^{(2)}:=\left(\begin{array}{cc}
k & \frac{\Delta_{\mathrm{s}}}{\ell_{\mathrm{s}}} z \\
\frac{\bar{\Delta}_{\mathrm{s}}}{\ell_{\mathrm{s}}} & -\bar{k}
\end{array}\right) .
$$

Jackiw and Rossi showed that Hamiltonian (6.1a) with a vortex in $\Delta_{\mathrm{s}}(z, \bar{z})$ satisfying $\ell_{\mathrm{s}}<\infty$ and $\left|\Delta_{\mathrm{s}}(z, \bar{z})\right|_{|z| \rightarrow \infty}<\infty$, supports one and only one bound state and that this bound state is pinned to the chemical potential, i.e., to the midgap of the BdG Hamiltonian. We will show explicitly that Hamiltonian (6.7), with an unbounded vortex profile, also has a singly degenerate solution, and thus there is one and only one Majorana fermion in the Rashba-Dirac limit at the Rashba-Dirac point if the pair potential is pure singlet. The stability of this Majorana fermion away from the RashbaDirac point or in the presence of a triplet pair potential is guaranteed by the fact that the particle-hole symmetry of the eigenvalue spectrum precludes the migration of the zero mode as long as the gap does not close. For large values of the chemical potential, it is natural to anticipate that many more bound states will have peeled off from the continuum with a level spacing à la Caroli-de-Gennes. This expectation is consistent with the computation from Ref. 61 of the states bound to a vortex as a function of the chemical potential for pure-singlet superconducting graphene.

Proof. Let

$$
c:=\frac{\Delta_{\mathrm{s}}}{\ell_{\mathrm{s}}} .
$$

We seek the solutions to

$$
0=\bar{k} u^{(1)}+c z v^{(1)},
$$




$$
0=\overline{c z} u^{(1)}-k v^{(1)}
$$

and

$$
\begin{aligned}
& 0=k u^{(2)}+c z v^{(2)}, \\
& 0=\overline{c z} u^{(2)}-\bar{k} v^{(2)},
\end{aligned}
$$

respectively. If we take the complex conjugate of the second condition in Eqs. (6.9a) and (6.9b), respectively, we get

$$
\begin{gathered}
0=\bar{k} u^{(1)}+c z v^{(1)}, \\
0=-\bar{k} \bar{v}^{(1)}+c z \bar{u}^{(1)},
\end{gathered}
$$

and

$$
\begin{gathered}
0=k u^{(2)}+c z v^{(2)}, \\
0=-k \bar{v}^{(2)}+c z \bar{u}^{(2)},
\end{gathered}
$$

respectively. For any $j=1,2$, if

$$
\left(\begin{array}{l}
u^{(j)}(k, \bar{k}) \\
v^{(j)}(k, \bar{k})
\end{array}\right)
$$

is a zero mode, so is

$$
\pm\left(\begin{array}{l}
\bar{v}^{(j)}(-k,-\bar{k}) \\
\bar{u}^{(j)}(-k,-\bar{k})
\end{array}\right)
$$

Hence, we try the ansatz

$$
\Phi_{ \pm}^{(j)}(k, \bar{k}):=\left(\begin{array}{c}
u^{(j)}(k, \bar{k}) \\
\pm \bar{u}^{(j)}(-k,-\bar{k})
\end{array}\right),
$$

where

$$
\begin{aligned}
0 & =\bar{k} u^{(1)}(k, \bar{k})+( \pm) c z \bar{u}^{(1)}(-k,-\bar{k}) \\
& =\kappa e^{-i \varphi} u^{(1)}(\kappa, \varphi)+( \pm) i c e^{+i \varphi}\left(\partial_{\kappa}+\frac{i}{\kappa} \partial_{\varphi}\right) \bar{u}^{(1)}(\kappa, \varphi+\pi)
\end{aligned}
$$

and

$$
\begin{aligned}
0 & =k u^{(2)}(k, \bar{k})+( \pm) c z \bar{u}^{(2)}(-k,-\bar{k}) \\
& =\kappa e^{i \varphi} u^{(2)}(\kappa, \varphi)+( \pm) i c e^{+i \phi}\left(\partial_{\kappa}+\frac{i}{\kappa} \partial_{\varphi}\right) \bar{u}^{(2)}(\kappa, \varphi+\pi)
\end{aligned}
$$

We choose a gauge in which

$$
\tilde{c}:=i c
$$

is real and make the ansatz

$$
\begin{gathered}
u_{ \pm}^{(1)}(\kappa, \varphi)=e^{i \varphi} w_{ \pm}^{(1)}(\kappa), \\
u_{ \pm}^{(2)}(\kappa, \varphi)=w_{ \pm}^{(2)}(\kappa),
\end{gathered}
$$

where the real-valued $w_{ \pm}^{(j)}(\kappa)$ satisfy

$$
0=\left[\kappa+(\mp) \widetilde{c}\left(\partial_{\kappa}+\frac{1}{\kappa}\right)\right] w_{ \pm}^{(1)}(\kappa)
$$

and

$$
0=\left[\kappa+( \pm) \widetilde{c} \partial_{\kappa}\right] w_{ \pm}^{(2)}(\kappa),
$$

respectively. The formal solutions to Eqs. (6.15b) and $(6.15 \mathrm{c})$ are

$$
w_{ \pm}^{(1)}(\kappa)=w_{ \pm}^{(1)}\left(\kappa_{0}\right) \exp \left\{\int_{\kappa_{0}}^{\kappa} d \kappa^{\prime}\left[( \pm) \frac{\kappa^{\prime}}{\widetilde{c}}-\frac{1}{\kappa^{\prime}}\right]\right\}
$$

and

$$
w_{ \pm}^{(2)}(\kappa)=w_{ \pm}^{(2)}\left(\kappa_{0}\right) \exp \left[-\int_{\kappa_{0}}^{\kappa} d \kappa^{\prime}( \pm) \frac{\kappa^{\prime}}{\tilde{c}}\right]
$$

respectively. Only

$$
w_{\operatorname{sgn} \widetilde{c}}^{(2)}(\kappa)=w_{\operatorname{sgn} \widetilde{c}}^{(2)}\left(\kappa_{0}\right) \exp \left(-\frac{\kappa^{2}-\kappa_{0}^{2}}{2|\widetilde{c}|}\right)
$$

is normalizable. We conclude that

$$
\Phi_{\operatorname{sgn} \tilde{c}}(\kappa, \varphi):=\left(\begin{array}{c}
1 \\
0 \\
0 \\
\operatorname{sgn} \tilde{c}
\end{array}\right) w_{\operatorname{sgn} \tilde{c}}^{(2)}(\kappa)
$$

is a Majorana state with the eigenvalue $\operatorname{sgn} \widetilde{c}$ under the particle-hole transformation (5.4). The uniqueness of this Majorana state, up to a normalization factor, can be proved along the same lines as is done in Appendix B.

\section{B. Fermi limit}

The Fermi limit is defined by the condition

$$
v_{\mathrm{RD}}=0 \text {. }
$$

In this limit and when the triplet-pair potential vanishes, isolated vortices support finite-energy Caroli-de-GennesMatricon bound states in the weak-coupling limit $\Delta_{\mathrm{s}} / \mu \ll 1$. No Majorana fermions are to be found tied to the core of an isolated vortex.

In the limit (6.18) with a vanishing singlet-pair potential,

$$
\mathcal{H}_{\text {vor }}=\left(\begin{array}{cccc}
\varepsilon(k, \bar{k}) & 0 & 0 & \frac{\Delta_{\mathrm{t}}}{2 \ell_{\mathrm{t}} k_{\mathrm{t}}}\{z, \bar{k}\} \\
0 & \varepsilon(k, \bar{k}) & \frac{\Delta_{\mathrm{t}}}{2 \ell_{\mathrm{t}} k_{\mathrm{t}}}\{z, k\} & 0 \\
0 & \text { H.c. } & -\varepsilon(k, \bar{k}) & 0 \\
\text { H.c. } & 0 & 0 & -\varepsilon(k, \bar{k})
\end{array}\right)
$$

decomposes into the direct sum of the $2 \times 2$ Hermitian operators 


$$
\mathcal{H}_{\mathrm{vor}}^{(1)}:=\left(\begin{array}{cc}
\varepsilon(k, \bar{k}) & \frac{\Delta_{\mathrm{t}}}{2 \ell_{\mathrm{t}} k_{\mathrm{t}}}\{z, \bar{k}\} \\
\frac{\bar{\Delta}_{\mathrm{t}}}{2 \ell_{\mathrm{t}} k_{\mathrm{t}}}\{z, \bar{k}\}^{\dagger} & -\varepsilon(k, \bar{k})
\end{array}\right)
$$

and

$$
\mathcal{H}_{\mathrm{vor}}^{(2)}:=\left(\begin{array}{cc}
\varepsilon(k, \bar{k}) & \frac{\Delta_{\mathrm{t}}}{2 \ell_{\mathrm{t}} k_{\mathrm{t}}}\{z, k\} \\
\frac{\bar{\Delta}_{\mathrm{t}}}{2 \ell_{\mathrm{t}} k_{\mathrm{t}}}\{z, k\}^{\dagger} & -\varepsilon(k, \bar{k})
\end{array}\right) .
$$

We claim that Hamiltonian $\mathcal{H}_{\text {vor }}$ supports two normalized zero modes if and only if (iff) the chemical potential $\mu>0$.

Proof. Define the short-hand notation

$$
c:=\frac{\Delta_{\mathrm{t}}}{\ell_{\mathrm{t}} k_{\mathrm{t}}} \in \mathrm{C} .
$$

We need

$$
\{z, \bar{k}\}=z \bar{k}+\bar{k} z=2 \bar{k} z+[z, \bar{k}]=2 \bar{k} z+2 i
$$

and

$$
\{z, \bar{k}\}^{\dagger}=k \bar{z}+\bar{z} k=2 k \bar{z}+[\bar{z}, k]=2 k \bar{z}+2 i .
$$

Equation (6.20) becomes

$$
\mathcal{H}_{\mathrm{vor}}^{(1)}=\left(\begin{array}{cc}
\varepsilon(k, \bar{k}) & c(\bar{k} z+i) \\
\bar{c}(k \bar{z}+i) & -\varepsilon(k, \bar{k})
\end{array}\right)
$$

and

$$
\mathcal{H}_{\mathrm{vor}}^{(2)}=\left(\begin{array}{cc}
\varepsilon(k, \bar{k}) & c k z \\
\bar{c} \bar{k} \bar{z} & -\varepsilon(k, \bar{k})
\end{array}\right) .
$$

We are going to show that operator (6.24a) has one and only one zero mode iff $\mu>0$. We will then show that the same is true for operator $(6.24 b)$.

We seek a solution to

$$
0=\mathcal{H}_{\mathrm{vor}}^{(1)}\left(\begin{array}{l}
u^{(1)} \\
v^{(1)}
\end{array}\right) .
$$

We must solve

$$
\begin{aligned}
& 0=\varepsilon(k, \bar{k}) u^{(1)}+c\left(\bar{k} 2 i \partial_{\bar{k}}+i\right) v^{(1)}, \\
& 0=\bar{c}\left(k 2 i \partial_{k}+i\right) u^{(1)}-\varepsilon(k, \bar{k}) v^{(1)} .
\end{aligned}
$$

If we take the complex conjugate of Eq. (6.26b), we get

$$
\begin{gathered}
0=\varepsilon(k, \bar{k}) u^{(1)}+c\left(\bar{k} 2 i \partial_{\bar{k}}^{-}+i\right) v^{(1)}, \\
0=-c\left(\bar{k} 2 i \partial_{\bar{k}}+i\right) \bar{u}^{(1)}-\varepsilon(k, \bar{k}) \bar{v}^{(1)} .
\end{gathered}
$$

We thus infer that a solution to Eq. (6.25), if it exists, is given by

$$
\Phi_{ \pm}^{(1)}=\left(\begin{array}{c}
u_{ \pm}^{(1)} \\
\pm \bar{u}_{ \pm}^{(1)}
\end{array}\right)
$$

where $u_{ \pm}^{(1)}$ is the solution to

$$
0=\varepsilon(k, \bar{k}) u_{ \pm}^{(1)}+( \pm) c\left(\bar{k} 2 i \partial_{\bar{k}}+i\right) \bar{u}_{ \pm}^{(1)} .
$$

Zero modes, if they exist, can be labeled by their angular momentum. We seek a zero mode with vanishing angular momentum, i.e., independent of $\varphi$. We must then solve

$$
\Phi_{ \pm}^{(1)}(\kappa)=\left(\begin{array}{c}
u_{ \pm}^{(1)}(\kappa) \\
\pm \bar{u}_{ \pm}^{(1)}(\kappa)
\end{array}\right), \quad 0 \leq \kappa<\infty
$$

where $u_{ \pm}^{(1)}$ is the solution to

$$
0=\varepsilon(\kappa) u_{ \pm}^{(1)}+( \pm)(i c)\left(\kappa \partial_{\kappa}+1\right) \bar{u}_{ \pm}^{(1)} .
$$

With the help of a global gauge transformation, we can always choose $c$ so that

$$
\widetilde{c}:=i c
$$

is real valued. Hence,

$$
0=\varepsilon(\kappa) u_{ \pm}^{(1)}(\kappa)+( \pm) \widetilde{c}\left(\kappa \partial_{\kappa}+1\right) \bar{u}_{ \pm}^{(1)}(\kappa)
$$

with $0 \leq \kappa<\infty$ admits a purely real or a purely imaginary solution since all coefficients of this first-order differential equation are real valued. We choose the real-valued solution. We divide Eq. (6.31) by $( \pm) \widetilde{c} \kappa$ to obtain the condition

$$
0=\left\{\partial_{\kappa}+\left[1+( \pm) \frac{\varepsilon(\kappa)}{\tilde{c}}\right] \frac{1}{\kappa}\right\} u_{ \pm}^{(1)}
$$

whose formal solution is given by

$$
u_{ \pm}^{(1)}(\kappa)=u_{ \pm}^{(1)}\left(\kappa_{0}\right) \exp \left\{-\int_{\kappa_{0}}^{\kappa} \frac{d \kappa^{\prime}}{\kappa^{\prime}}\left[1+( \pm) \frac{\varepsilon\left(\kappa^{\prime}\right)}{\tilde{c}}\right]\right\} .
$$

The formal solution $(6.32 \mathrm{~b})$ is admissible iff it is normalizable, i.e., if

$$
\int_{0}^{\infty} d \kappa \kappa\left|u_{ \pm}^{(1)}(\kappa)\right|^{2}<\infty
$$

Define

$$
\begin{aligned}
F_{ \pm}(\kappa) & :=\int_{\kappa_{0}}^{\kappa} \frac{d \kappa^{\prime}}{\kappa^{\prime}}\left[1+( \pm) \frac{\varepsilon\left(\kappa^{\prime}\right)}{\widetilde{c}}\right] \\
& =\left[1-( \pm) \frac{\mu}{\widetilde{c}}\right] \ln \frac{\kappa}{\kappa_{0}}+( \pm) \frac{\kappa^{2}-\kappa_{0}^{2}}{4 m \widetilde{c}} .
\end{aligned}
$$

For large $\kappa$,

$$
F_{ \pm}(\kappa) \sim( \pm) \frac{\kappa^{2}}{4 m \tilde{c}}
$$

so that normalizability imposes the choice

$$
\pm=\operatorname{sgn} \tilde{c}
$$

and the formal solution (6.32b) becomes 


$$
u_{\mathrm{sgn} \tilde{c}}^{(1)}(\kappa)=u_{\mathrm{sgn} \tilde{c}}^{(1)}\left(\kappa_{0}\right) \times\left(\frac{\kappa}{\kappa_{0}}\right)^{(\mu / \mid \vec{c})-1} \times e^{-\left(\kappa^{2}-\kappa_{0}^{2}\right) / 4 m|\widetilde{c}|} .
$$

For small $\kappa$,

$$
F_{\text {sgn } \widetilde{c}}(\kappa) \sim\left(1-\frac{\mu}{|\widetilde{c}|}\right) \ln \frac{\kappa}{\kappa_{0}}
$$

so that normalizability demands convergence of

$$
\int_{0}^{\kappa_{0}} d \kappa \kappa^{2(\mu|| \widetilde{c})-1}
$$

i.e.,

$$
\mu>0 .
$$

We conclude that for any choice of gauge such that $\widetilde{c} \equiv i c$ is real valued

$$
\Phi_{\operatorname{sgn} \widetilde{c}}^{(1)}(\kappa)=\left(\begin{array}{c}
u_{\operatorname{sgn} \widetilde{c}}^{(1)}(\kappa) \\
(\operatorname{sgn} \widetilde{c}) \bar{u}_{\operatorname{sgn} \tilde{c}}^{(1)}(\kappa)
\end{array}\right), \quad 0 \leq \kappa<\infty,
$$

where

$$
u_{\mathrm{sgn} \tilde{c}}^{(1)}(\kappa)=u_{\mathrm{sgn} \tilde{c}}^{(1)}\left(\kappa_{0}\right) \times\left(\frac{\kappa}{\kappa_{0}}\right)^{(\mu / \mid \tilde{c})-1} \times e^{-\left(\kappa^{2}-\kappa_{0}^{2}\right) / 4 m|\widetilde{c}|}
$$

is a normalizable Majorana mode iff $\mu>0$. Observe that $\Phi_{\operatorname{sgn} c}^{(1)}(k)$ is an eigenstate with the eigenvalue $-\operatorname{sgn} \widetilde{c}$ of the particle-hole transformation defined in Eq. (5.4), i.e.,

$$
X_{22} \Phi_{\mathrm{sgn} \tilde{c}}^{(1) *}(-\boldsymbol{k})=-\operatorname{sgn} \widetilde{c} \Phi_{\mathrm{sgn} \tilde{c}}^{(1)}(\boldsymbol{k}) .
$$

To show that solution (6.41) is unique, up to a normalization, one expands Eq. (6.25) in polar harmonics labeled by the angular quantum number $n \in \mathbb{Z}$ (see Appendix B). Modes with angular quantum number $\pm n$ are pairwise coupled. A formal zero mode of the form Eq. (6.41) whereby the function $u$ is substituted by a doublet, i.e.,

$$
\mathrm{U}_{ \pm, n}^{(1)}(\kappa)=\exp \left[-\mathrm{F}_{ \pm, n}^{(1)}(\kappa)\right] \mathrm{U}_{ \pm, n}^{(1)}\left(\kappa_{0}\right)
$$

with

$$
\mathbb{F}_{ \pm, n}^{(1)}(\kappa)=\int_{\kappa_{0}}^{\kappa} \frac{d \kappa^{\prime}}{\kappa^{\prime}} G_{ \pm, n}^{(1)}\left(\kappa^{\prime}\right)
$$

and $\mathrm{G}_{ \pm, n}^{(1)}\left(\kappa^{\prime}\right)$ both $2 \times 2$ matrices, follows. However, it is not normalizable.

It is time to seek a solution to

$$
0=\mathcal{H}_{\mathrm{vor}}^{(2)}\left(\begin{array}{l}
u^{(2)} \\
v^{(2)}
\end{array}\right) \text {. }
$$

We must solve

$$
0=\varepsilon(k, \bar{k}) u^{(2)}+c k 2 i \partial_{\bar{k}} v^{(2)}
$$

$$
0=\bar{c} \bar{k} 2 i \partial_{k} u^{(2)}-\varepsilon(k, \bar{k}) v^{(2)} .
$$

If we take the complex conjugate of Eq. (6.45b), we get

$$
\begin{gathered}
0=\varepsilon(k, \bar{k}) u^{(2)}+c k 2 i \partial_{\bar{k}} v^{(2)}, \\
0=-c k 2 i \partial_{\bar{k}} \bar{u}^{(2)}-\varepsilon(k, \bar{k}) \bar{v}^{(2) .}
\end{gathered}
$$

We thus infer that a solution to Eq. (6.44), if it exists, is given by

$$
\Phi_{ \pm}^{(2)}=\left(\begin{array}{c}
u_{ \pm}^{(2)} \\
\pm \bar{u}_{ \pm}^{(2)}
\end{array}\right)
$$

where $u_{ \pm}^{(2)}$ is the solution to

$$
0=\varepsilon(\kappa) u_{ \pm}^{(2)}+( \pm) \widetilde{c} \kappa e^{2 i \varphi}\left(\partial_{\kappa}+\frac{i}{\kappa} \partial_{\varphi}\right) \bar{u}_{ \pm}^{(2)} .
$$

When expanding the solution in angular momentum modes $\exp (\operatorname{in} \varphi), n \in \mathbb{Z}$, the mode $n=1$ turns out to be the only mode that does not couple to other modes via Eq. (6.47b). The ansatz

$$
u_{\mathrm{sgn} \tilde{c}}^{(2)}(\kappa, \varphi)=e^{i \varphi} w_{\mathrm{sgn} \tilde{c}}^{(2)}(\kappa)
$$

casts Eq. (6.47b) in the same form as Eq. (6.31) so that

$$
w_{\operatorname{sgn} \tilde{c}}^{(2)}(\kappa)=w_{\operatorname{sgn} \tilde{c}}^{(2)}\left(\kappa_{0}\right) \times\left(\frac{\kappa}{\kappa_{0}}\right)^{(\mu / \tilde{c})-1} \times e^{-\left(\kappa^{2}-\kappa_{0}^{2}\right) / 4 m|\tilde{c}|} .
$$

Observe that $\Phi_{\operatorname{sgn} \tilde{c}}^{(2)}(\boldsymbol{k})$ with $u_{ \pm}^{(2)}(\boldsymbol{k})$ given in Eq. (6.48) is an eigenstate with the eigenvalue $-\operatorname{sgn} \widetilde{c}$ of the particle-hole transformation defined in Eq. (5.4), i.e.,

$$
X_{22} \Phi_{\operatorname{sgn} \tilde{c}}^{(2) *}(-k)=-\operatorname{sgn} \widetilde{c} \Phi_{\operatorname{sgn} \tilde{c}}^{(2)}(k) .
$$

It remains to verify that the spinor (6.8) is single valued in real space, i.e., that the Fourier transform of the function (6.48a) vanishes at the origin of the complex$z=r \exp (i \theta)$ plane. Hence, we need the small $r$ expansion of the Fourier transform

$$
\begin{aligned}
u_{\mathrm{sgn} \tilde{c}}^{(2)}(r, \theta) & \propto \int_{0}^{\infty} d \kappa \kappa \int_{0}^{2 \pi} d \varphi e^{i r \kappa \cos (\varphi-\theta)} \times e^{i \varphi} w_{\mathrm{sgn} \tilde{c}}^{(2)}(\kappa) \\
& =e^{i \theta} \int_{0}^{\infty} d \kappa \kappa \int_{0}^{2 \pi} d \phi e^{i r \kappa \cos \phi} \times e^{i \phi} w_{\mathrm{sgn} \tilde{c}}^{(2)}(\kappa) .
\end{aligned}
$$

The $\kappa$ integral is well behaved for large $\kappa$ because of the Gaussian factor. Moreover, an upper cutoff to this integral can be used up to Gaussian accuracy. If so, a Taylor expansion of $\exp ($ ir $\kappa \cos \phi)$ in the integrand can be performed to capture the leading dependence on $r$. The integral over $\phi$ eliminates the term independent of $r$ so that

$$
u_{\text {sgn } \vec{c}}(r, \theta) \sim r e^{i \theta}+\mathcal{O}\left(r^{2}\right)
$$

is single valued at the origin $r=0$ and thus an admissible solution. 
We conclude that there are two Majorana states

$$
\Phi_{\operatorname{sgn} \tilde{c}}(\kappa, \varphi)=\left[A\left(\begin{array}{c}
1 \\
0 \\
0 \\
\operatorname{sgn} \tilde{c}
\end{array}\right)+B\left(\begin{array}{c}
0 \\
e^{i \varphi} \\
\operatorname{sgn} \tilde{c} e^{-i \varphi} \\
0
\end{array}\right)\right] u_{\operatorname{sgn} \tilde{c}(\kappa)}
$$

( $A$ and $B$ are real numbers) bound to the core of an isolated vortex satisfying the linear profile (6.5) in the triplet-pair potential. The Majorana state weighted by the coefficient $A$ is related to the Majorana state weighted by the coefficient $B$ through the helical symmetry defined in Eq. (5.5) and not by the operation of time reversal defined in Eq. (5.6). This is expected since TRS is broken by the vortex.

$\mathrm{Lu}$ and Yip in Ref. 44 (see also Sato and Fujimoto in Ref. 45) also found two Majorana fermions bound to the core of a vortex with unit vorticity in a weakly coupled (i.e., a large chemical potential compared to the pairing potentials) 2D TRS noncentrosymmetric superconductor with dominant triplet-pair potential. Their first zero mode is the real-space counterpart to the mode (6.41). Their second zero mode carries angular momentum $n=1$ and is the real-space counterpart to the mode (6.48).

In Ref. 32, Qi et al. have studied zero modes bound to the core of vortices in TRS $p_{x} \pm i p_{y}$ superconductors as well. Viewing the system as a combination of a $p_{x}+i p_{y}$ superfluid (which corresponds to $\mathcal{H}_{\text {vor }}^{(1)}$ ) and its time-reversed partner, a $p_{x}-i p_{y}$ superfluid (which corresponds to $\mathcal{H}_{\text {vor }}^{(2)}$ ), they simultaneously introduced a vortex in the former and an antivortex in the latter. In contrast to our study of a TRS-breaking vortex, this configuration of a pair of vortex and antivortex is TRS and the two Majorana modes obtained by Qi et al. are connected by the operation of time reversal. Whereas the Majorana fermions (6.52) are not robust to a generic perturbation that breaks translation invariance, the TRS-protected pair of Majorana fermions obtained by Qi et al. is robust to any weak perturbation that preserves TRS.

\section{Away from the Rashba-Dirac and Fermi limits}

Majorana fermions tied to vortices are robust to continuous changes in the BdG Hamiltonian as long as the spectral gap does not close, for all nonvanishing energy eigenvalues occur pairwise with the energies $\pm E$ owing to the particlehole symmetry (5.4). There will be one (two) Majorana fermion(s) tied to the core of an isolated vortex carrying vorticity one in regions I (II) of Fig. 3 (Fig. 4). By the same reasoning, region III of Fig. 4 does not admit Majorana fermions bound to the core of unit vortices. ${ }^{62}$ Regions II and III in Fig. 4 differ by the even number of Majorana fermions that TRS-breaking vortices can accommodate. This distinction is not robust to any generic perturbation that breaks translation symmetry, for it is not protected by TRS.

\section{DISCUSSION}

In this paper, we studied the possible superconducting phases of the surface states of 3D TRS topological insulators and 2D TRS noncentrosymmetric metals. Both systems share remarkable magnetoelectric effects, however their bulk superconducting phases differ in important ways. The difference stems from the topology of the bands. Surface states of 3D TRS topological insulators are topologically equivalent to a single species of Rashba-Dirac fermions while noncentrosymmetric metals are Fermi like with two Fermi surfaces for large chemical potentials. As a result, we find that there is a unique superconducting phase in the case of the RashbaDirac limit while there are two phases in the Fermi limit.

We studied the phase diagram as a function of the strengths of the mean-field pair potentials $\Delta_{\mathrm{s}}$ (singlet) and $\Delta_{\mathrm{t}}$ (triplet), as well as $\mu$ (chemical potential). In the RashbaDirac limit, a single Majorana fermion is bound to the core of an isolated and TRS-breaking vortex with unit winding number in the superconducting order parameter everywhere in the phase diagram in the $\Delta_{\mathrm{t}} / \Delta_{\mathrm{s}}-\mu$ plane, with the exceptions where the gap closes. The gap-closing lines do not separate distinct phases in the Rashba-Dirac limit, because one can always connect two sides of a gap-closing line by, instead of crossing the line directly, going through the point at infinity $\left(\Delta_{\mathrm{s}}=0\right)$ without closing the gap. Evidently, gap closing is a necessary but not sufficient condition to have two distinct phases.

In the Fermi limit, we find instead that there are two superconducting phases. These phases correspond to singlet or triplet dominated physics. In the singlet-dominated phase, we find that an isolated TRS-breaking vortex with unit winding number (a full vortex) does not bind Majorana fermions. In the triplet-dominated phase, we find a pair of Majorana fermions bound to an isolated full vortex. Hence, these Majorana states have a distinct origin from those found for half vortices in the $p_{x} \pm i p_{y}$ superconductors. The physical reason for this difference is that TRS forces the spin-resolved pairing amplitudes $\Delta_{\uparrow \uparrow}$ and $\Delta_{\downarrow \downarrow}$ to be related, and thus one cannot introduce vorticity in one but not the other, as can be done with half vortices in the $p_{x} \pm i p_{y}$ superconductors.

\section{ACKNOWLEDGMENTS}

We thank S. Ryu and A. Furusaki for many useful discussions. This work was supported in part by the DOE under Grant No. DE-FG02-06ER46316 (C.C.). C.M. thanks the Condensed Matter Theory Visitor's Program at Boston University for support. T.N. acknowledges the German National Academic Foundation for financial support.

\section{APPENDIX A: PROOF OF Eqs. (4.4), (4.9), and (4.13)}

All reduced interaction Hamiltonians (4.3), (4.8), and (4.12) have summands which can be represented in terms of the helicity basis using the transformation $(2.4 \mathrm{~d})$. To do this explicitly, let $\mu$ and $\nu$ run from 0 to 3 with $\sigma_{0}$ the $2 \times 2$ unit matrix and write

$$
\begin{aligned}
& \left(\psi_{\boldsymbol{k}}^{\dagger} \sigma^{(\mu)} \psi_{\boldsymbol{p}}\right)\left(\psi_{-\boldsymbol{k}}^{\dagger} \sigma^{(\nu)} \psi_{-\boldsymbol{p}}\right) \\
& \quad=\sum_{s_{1}, s_{2}, s_{3}, s_{4}} \sigma_{s_{1}, s_{4}}^{(\mu)} \sigma_{s_{2}, s_{3}}^{(\nu)} c_{\boldsymbol{k} s_{1}}^{\dagger} c_{-\boldsymbol{k} s_{2}}^{\dagger} c_{-p s_{3}} c_{p s_{4}}+\cdots \\
& \quad=\left(\tilde{\psi}_{\boldsymbol{k}}^{\dagger} \Pi_{\boldsymbol{k}}^{\dagger} \sigma^{(\mu)} \Pi_{\boldsymbol{p}} \tilde{\psi}_{\boldsymbol{p}}\right)\left(\tilde{\psi}_{-\boldsymbol{k}}^{\dagger} \Pi_{-\boldsymbol{k}}^{\dagger} \sigma^{(\nu)} \Pi_{-\boldsymbol{p}} \tilde{\psi}_{-\boldsymbol{p}}\right) .
\end{aligned}
$$


The $\cdots$ stands for two-fermion contributions that result from anticommuting the operators.

We first evaluate the matrix products,

$$
\begin{aligned}
\Pi_{ \pm k}^{\dagger} \sigma^{(0)} \Pi_{ \pm p}= & \exp \left(i \frac{\varphi_{p}-\varphi_{k}}{2}\right)\left[\cos \frac{\varphi_{p}-\varphi_{k}}{2} \sigma^{(0)}\right. \\
& \left.-i \sin \frac{\varphi_{p}-\varphi_{k}}{2} \sigma^{(1)}\right] \\
\Pi_{ \pm k}^{\dagger} \sigma^{(1)} \Pi_{ \pm p}= & \pm \exp \left(i \frac{\varphi_{p}-\varphi_{k}}{2}\right)\left[\cos \frac{\varphi_{p}+\varphi_{k}}{2} \sigma^{(3)}\right. \\
& \left.+\sin \frac{\varphi_{p}+\varphi_{k}}{2} \sigma^{(2)}\right], \\
\Pi_{ \pm k}^{\dagger} \sigma^{(2)} \Pi_{ \pm p}= & \mp \exp \left(i \frac{\varphi_{p}-\varphi_{k}}{2}\right)\left[\cos \frac{\varphi_{p}+\varphi_{k}}{2} \sigma^{(2)}\right. \\
& \left.-\sin \frac{\varphi_{p}+\varphi_{k}}{2} \sigma^{(3)}\right], \\
& \left.-i \sin \frac{\varphi_{p}-\varphi_{k}}{2} \sigma^{(0)}\right] . \\
\Pi_{ \pm k}^{\dagger} \sigma^{(3)} \Pi_{ \pm p}= & \exp \left(i \frac{\varphi_{p}-\varphi_{k}}{2}\right)\left[\cos \frac{\varphi_{p}-\varphi_{k}}{2} \sigma^{(1)}\right.
\end{aligned}
$$

Here, we observe that any of the right-hand sides in Eq. (A2) involves one diagonal and one off-diagonal Pauli matrix. At this point we can partly settle our constraint to have only Cooper pairs made of electrons of the same helicity. For this case, only products of two diagonal or two off-diagonal terms contribute in the product, Eq. (A1).

Second, we introduce the notation

$$
\eta_{k \lambda \mid p \lambda^{\prime}}:=a_{k \lambda}^{\dagger} a_{-k \lambda}^{\dagger} a_{-p \lambda^{\prime}} a_{p \lambda^{\prime}}, \quad \lambda, \lambda= \pm,
$$

in terms of which we find

$$
\begin{aligned}
& \tilde{\psi}_{\boldsymbol{k}}^{\dagger} \sigma^{(0)} \widetilde{\psi}_{\boldsymbol{p}} \tilde{\psi}_{-\boldsymbol{k}}^{\dagger} \sigma^{(0)} \tilde{\psi}_{-p}=+\widetilde{\psi}_{\boldsymbol{k}}^{\dagger} \sigma^{(3)} \tilde{\psi}_{\boldsymbol{p}} \tilde{\psi}_{-\boldsymbol{k}}^{\dagger} \sigma^{(3)} \tilde{\psi}_{-p} \\
& =\eta_{k+\mid p+}+\eta_{k-\mid p-}+\cdots, \\
& \tilde{\psi}_{\boldsymbol{k}}^{\dagger} \sigma^{(1)} \tilde{\psi}_{\boldsymbol{p}} \tilde{\psi}_{-\boldsymbol{k}}^{\dagger} \sigma^{(1)} \tilde{\psi}_{-p}=-\widetilde{\psi}_{\boldsymbol{k}}^{\dagger} \sigma^{(2)} \tilde{\psi}_{\boldsymbol{p}} \tilde{\psi}_{-\boldsymbol{k}}^{\dagger} \sigma^{(2)} \tilde{\psi}_{-p} \\
& =\eta_{k+\mid p-}+\eta_{k-\mid p+}+\cdots, \\
& \tilde{\psi}_{\boldsymbol{k}}^{\dagger} \sigma^{(0)} \tilde{\psi}_{\boldsymbol{p}} \tilde{\psi}_{-\boldsymbol{k}}^{\dagger} \sigma^{(3)} \tilde{\psi}_{-\boldsymbol{p}}=+\tilde{\psi}_{\boldsymbol{k}}^{\dagger} \sigma^{(3)} \tilde{\psi}_{\boldsymbol{p}} \tilde{\psi}_{-\boldsymbol{k}}^{\dagger} \sigma^{(0)} \tilde{\psi}_{-\boldsymbol{p}} \\
& =\eta_{k+\mid p+}-\eta_{k-\mid p-}+\cdots, \\
& \tilde{\psi}_{k}^{\dagger} \sigma^{(1)} \tilde{\psi}_{p} \tilde{\psi}_{-k}^{\dagger} \sigma^{(2)} \tilde{\psi}_{-p}=+\tilde{\psi}_{k}^{\dagger} \sigma^{(2)} \tilde{\psi}_{\boldsymbol{p}} \tilde{\psi}_{-k}^{\dagger} \sigma^{(1)} \tilde{\psi}_{-p} \\
& =-i\left(\eta_{k+\mid p-}-\eta_{k-\mid p+},\right)+\cdots \text {. }
\end{aligned}
$$

Here, $\cdots$ stands for contributions which would lead to Cooper pairs made up of two electrons of different helicity. We are left with the task of collecting the phase and trigonometric multiplicative factors from Eq. (A2).

For the density-density interaction (4.3) we have to compute Eq. (A1) with $\mu=\nu=0$. According to Eq. (A2) this involves collecting the phase and trigonometric multiplicative factors for Eqs. (A4a) and (A4b). We find

$$
\begin{aligned}
H_{V}^{\mathrm{red}} & =\frac{1}{2} \sum_{k, p} V_{k-p}\left(\widetilde{\psi}_{k}^{\dagger} \Pi_{k}^{\dagger} \Pi_{p} \widetilde{\psi}_{p}\right)\left(\widetilde{\psi}_{-k}^{\dagger} \Pi_{-k}^{\dagger} \Pi_{-p} \tilde{\psi}_{-p}\right) \\
& =2 \sum_{k p} V_{k-p} e^{i\left(\varphi_{p}-\varphi_{k}\right)}\left[\cos ^{2} \frac{\varphi_{p}-\varphi_{k}}{2}\left(\eta_{k+\mid p+}+\eta_{k-\mid p-}\right)-\sin ^{2} \frac{\varphi_{p}-\varphi_{k}}{2}\left(\eta_{k+\mid p-}+\eta_{k-\mid p+}\right)\right]
\end{aligned}
$$

from which Eq. (4.4) follows.

For the Heisenberg interaction (4.8) we have to compute Eq. (A1) with $\mu=\nu=1,2,3$. According to Eq. (A2) this involves collecting the phase and trigonometric multiplicative factors for Eqs. (A4a) and (A4b). We find

$$
\begin{aligned}
H_{\mathrm{H}}^{\mathrm{red}} & =\frac{1}{8} \sum_{k, p} \sum_{j=1}^{3} J_{k-p}\left(\widetilde{\psi}_{k}^{\dagger} \Pi_{k}^{\dagger} \sigma^{(j)} \Pi_{p} \tilde{\psi}_{p}\right)\left(\widetilde{\psi}_{-k}^{\dagger} \Pi_{-k}^{\dagger} \sigma^{(j)} \Pi_{-p} \tilde{\psi}_{-p}\right) \\
& =\frac{1}{8} \sum_{k, p} J_{k-p} e^{i\left(\varphi_{p}-\varphi_{k}\right)}\left[\eta_{k+\mid p-}+\eta_{k-\mid p+}-\eta_{k+\mid p+}-\eta_{k-\mid p-}+\cos ^{2} \frac{\varphi_{p}-\varphi_{k}}{2}\left(\eta_{k+\mid p-}+\eta_{k-\mid p+}\right)-\sin ^{2} \frac{\varphi_{p}-\varphi_{k}}{2}\left(\eta_{k+\mid p+}+\eta_{k-\mid p-}\right)\right]
\end{aligned}
$$

from which Eq. (4.9) follows.

Finally, the Dzyaloshinskii-Moriya interaction (4.12) involves terms of the type (A1) with $(\mu, \nu)=(2,3),(3,2)$, $(1,3)$, and $(3,1)$ which in turn lead to Eqs. (A4c) and (A4d). The calculations yields 


$$
\begin{aligned}
H_{\mathrm{DM}}^{\mathrm{red}}= & \frac{1}{8} \sum_{k, p} \sum_{j, l=1, \ldots 3}^{m=1,2} \epsilon_{j l m} D_{k-p}^{(m)}\left(\widetilde{\psi}_{k}^{\dagger} \Pi_{k}^{\dagger} \sigma^{(j)} \Pi_{p} \tilde{\psi}_{p}\right)\left(\widetilde{\psi}_{-k}^{\dagger} \Pi_{-k}^{\dagger} \sigma^{(l)} \Pi_{-p} \tilde{\psi}_{-p}\right) \\
= & \frac{i}{4} \sum_{k, p} e^{i\left(\varphi_{p}-\varphi_{k}\right)}\left[\left(\eta_{k+\mid p-}-\eta_{k-\mid p+}\right)\left(D_{k-p}^{(1)} \cos \frac{\varphi_{p}+\varphi_{k}}{2} \cos \frac{\varphi_{p}-\varphi_{k}}{2}+D_{k-p}^{(2)} \sin \frac{\varphi_{p}+\varphi_{k}}{2} \cos \frac{\varphi_{p}-\varphi_{k}}{2}\right)\right. \\
& \left.-\left(\eta_{k+\mid p+}-\eta_{k-\mid p-}\right)\left(D_{k-p}^{(1)} \sin \frac{\varphi_{p}+\varphi_{k}}{2} \sin \frac{\varphi_{p}-\varphi_{k}}{2}-D_{k-p}^{(2)} \cos \frac{\varphi_{p}+\varphi_{k}}{2} \sin \frac{\varphi_{p}-\varphi_{k}}{2}\right)\right]
\end{aligned}
$$

from which Eq. (4.13) follows.

\section{APPENDIX B: UNNORMALIZABILITY OF HIGHER ANGULAR MOMENTUM ZERO MODES}

We are going to show that the solutions to Eq. (6.28b) with nonzero angular momentum and the solutions to Eq. (6.47b) with angular momentum other than +1 are not normalizable. We expand

$$
\begin{aligned}
u_{ \pm}^{(1)}(\kappa, \varphi) & =\sum_{n \geq 0}\left[e^{i n \varphi} f_{ \pm, n}^{(1)}(\kappa)+e^{-i n \varphi} g_{ \pm, n}^{(1)}(\kappa)\right], \\
u_{ \pm}^{(2)}(\kappa, \varphi) & =\sum_{n \geq-1}\left[e^{i(n+2) \varphi} f_{ \pm, n}^{(2)}(\kappa)+e^{-i n \varphi} g_{ \pm, n}^{(2)}(\kappa)\right],
\end{aligned}
$$

for Eqs. (6.28b) and (6.47b), respectively. The differential equations mutually couples two and only two angular momentum modes. As all coefficients of the differential equation are purely real, the expansion parameters $f_{ \pm, n}^{(j)}(\kappa)$ and $g_{ \pm, n}^{(j)}(\kappa)$ can be chosen to be either purely real or purely imaginary numbers. Without loss of generality, we make the former choice. In terms of the doublet $\mathrm{U}_{ \pm, n}^{(j)}=\left[f_{ \pm, n}^{(j)}, g_{ \pm, n}^{(j)}\right]^{T}$ that represents the two coupled modes labeled by $n$, we find

$$
\partial_{\kappa} \mathrm{U}_{ \pm, n}^{(j)}(\kappa)=-\frac{1}{\kappa} \mathrm{G}_{ \pm, n}^{(j)}(\kappa) \mathrm{U}_{ \pm, n}^{(j)}(\kappa)
$$

The matrices are given by

$$
G_{ \pm, n}^{(j)}(\kappa)=\left(\begin{array}{cc}
2-(j+n) & \pm \frac{\varepsilon(\kappa)}{\tilde{c}} \\
\pm \frac{\varepsilon(\kappa)}{\tilde{c}} & j+n
\end{array}\right)
$$

Hence, the doublet solution can be written as

$$
\mathrm{U}_{ \pm, n}^{(j)}(\kappa)=\exp \left[-\mathbb{F}_{ \pm, n}^{(j)}(\kappa)\right] \mathrm{U}_{ \pm, n}^{(j)}\left(\kappa_{0}\right)
$$

with

$$
\mathbb{F}_{ \pm, n}^{(j)}(\kappa)=\int_{\kappa_{0}}^{\kappa} \frac{d \kappa^{\prime}}{\kappa^{\prime}} \mathrm{G}_{ \pm, n}^{(j)}(\kappa) .
$$

The demand of normalizability reads

$$
\begin{aligned}
& \int_{0}^{\infty} d \kappa \kappa\left[\mathrm{U}_{ \pm, n}^{(j)}(\kappa)\right]^{T} \mathrm{U}_{ \pm, n}^{(j)}(\kappa) \\
& \quad=\int_{0}^{\infty} d \kappa \kappa\left[\mathrm{U}_{ \pm, n}^{(j)}\left(\kappa_{0}\right)\right]^{T} \exp \left[-2 \mathbb{F}_{ \pm, n}^{(j)}(\kappa)\right] \mathrm{U}_{ \pm, n}^{(j)}\left(\kappa_{0}\right)<\infty
\end{aligned}
$$

In the limit of large $\kappa$, both matrices $\mathrm{G}_{ \pm, n}^{(j)}(\kappa)(j=1,2)$ obey the same behavior. The matrix in the exponent becomes for both cases $j=1,2$,

$$
\mathbb{F}_{ \pm, n}^{(j)}(\kappa) \rightarrow \pm \frac{\kappa^{2}}{2 m \tilde{c}} \sigma_{1}
$$

Upon exponentiation it, the condition (B4) then reads

$$
\begin{gathered}
\int_{0}^{\infty} d \kappa \kappa\left\{\cosh \left(\frac{\kappa^{2}}{m \tilde{c}}\right)\left\{\left[f_{ \pm, n}^{(j)}\left(\kappa_{0}\right)\right]^{2}+\left[g_{ \pm, n}^{(j)}\left(\kappa_{0}\right)\right]^{2}\right\}\right. \\
\left.\mp 2 \sinh \left(\frac{\kappa^{2}}{m \tilde{c}}\right) f_{ \pm, n}^{(j)}\left(\kappa_{0}\right) g_{ \pm, n}^{(j)}\left(\kappa_{0}\right)\right\}<\infty,
\end{gathered}
$$

which gives a condition for the initial values

$$
f_{ \pm, n}^{(j)}\left(\kappa_{0}\right)= \pm \operatorname{sgn} \widetilde{c} g_{ \pm, n}^{(j)}\left(\kappa_{0}\right)
$$

In the opposite limit of small $\kappa$, we find for the matrix that has to be exponentiated

$$
\mathbb{F}_{ \pm, n}^{(j)}(\kappa) \rightarrow \mathrm{G}_{ \pm, n}^{(j)}(0) \ln \frac{\kappa}{\kappa_{0}}
$$

Upon exponentiating it, the condition (B4) reads 


$$
\begin{aligned}
\infty> & \int_{0}^{\infty} d \kappa \kappa \frac{\left.\kappa^{-2\left[1+\sqrt{(n+j-1)^{2}+\left(\mu^{2} / \tilde{c}^{2}\right)}\right.}\right]}{\sqrt{(n+j-1)^{2}+\frac{\mu^{2}}{\widetilde{c}^{2}}}}\left\{\left[f_{ \pm, n}^{(j)}\left(\kappa_{0}\right)\right]^{2}+\left[g_{ \pm, n}^{(j)}\left(\kappa_{0}\right)\right]^{2}\right\} \sqrt{(n+j-1)^{2}+\frac{\mu^{2}}{\tilde{c}^{2}}} \\
& +\left\{\left[f_{ \pm, n}^{(j)}\left(\kappa_{0}\right)\right]^{2}-\left[g_{ \pm, n}^{(j)}\left(\kappa_{0}\right)\right]^{2}\right\}(n+j-1) \mp 2 \frac{\mu}{\widetilde{c}} f_{ \pm, n}^{(j)}\left(\kappa_{0}\right) g_{ \pm, n}^{(j)}\left(\kappa_{0}\right)+\mathcal{O}\left(\kappa^{\left.4 \sqrt{(n+j-1)^{2}+\left(\mu^{2} / \tilde{c}^{2}\right)}\right)}\right\} .
\end{aligned}
$$

All terms that are given explicitly in the curly bracket have to vanish in order to achieve normalizability. This amounts to

$$
f_{ \pm, n}^{(j)}\left(\kappa_{0}\right)= \pm \frac{\tilde{c}}{\mu}\left[\sqrt{(n+j-1)^{2}+\frac{\mu^{2}}{\widetilde{c}^{2}}}-(n+j-1)\right] g_{ \pm, n}^{(j)}\left(\kappa_{0}\right) .
$$

Both conditions (B7) and (B10) are only satisfied simultaneously if $n=1-j$. For this mode not to be vanishing, the sign in Eq. (B7) must be chosen $\pm=\operatorname{sgn} \tilde{c}$, which is only compatible with Eq. (B10) for $\mu>0$. This corresponds to the solutions discussed in Sec. VI and we conclude that these are the only normalizable zero modes for each of the blocks $j=1,2$.

${ }^{1}$ E. Mooser and W. B. Pearson, Phys. Rev. 101, 492 (1956).

${ }^{2}$ J. Black, E. M. Conwell, L. Seigle, and C. W. Spencer, J. Phys. Chem. Solids 2, 240 (1957).

${ }^{3}$ Y. Xia, D. Qian, D. Hsieh, L. Wray, A. Pal, H. Lin, A. Bansil, D. Grauer, Y. S. Hor, R. J. Cava, and M. Z. Hasan, Nat. Phys. 5, 398 (2009).

${ }^{4}$ H. Zhang, C.-X. Liu, X.-L. Qi, X. Dai, Z. Fang, and S.-C. Zhang, Nat. Phys. 5, 438 (2009).

${ }^{5}$ D. Hsieh, D. Qian, L. Wray, Y. Xia, Y. S. Hor, R. J. Cava, and M. Z. Hasan, Nature (London) 452, 970 (2008).

${ }^{6}$ D. Hsieh, Y. Xia, L. Wray, D. Qian, A. Pal, J. H. Dil, J. Osterwalder, F. Meier, G. Bihlmayer, C. L. Kane, Y. S. Hor, R. J. Cava, and M. Z. Hasan, Science 323, 919 (2009).

${ }^{7}$ L. Fu, C. L. Kane, and E. J. Mele, Phys. Rev. Lett. 98, 106803 (2007).

${ }^{8}$ J. E. Moore and L. Balents, Phys. Rev. B 75, 121306(R) (2007).

${ }^{9}$ R. Roy, Phys. Rev. B 79, 195321 (2009).

${ }^{10}$ Y. Hor, A. Williams, J. Checkelsky, P. Roushan, J. Seo, Q. Xu, H. Zandbergen, A. Yazdani, N. Ong, and R. Cava, Phys. Rev. Lett. 104, 057001 (2010).

${ }^{11}$ For a general review on graphene, see A. H. Castro Neto, F. Guinea, N. M. R. Peres, K. S. Novoselov, and A. K. Geim, Rev. Mod. Phys. 81, 109 (2009).

${ }^{12}$ P. M. Ostrovsky, I. V. Gornyi, and A. D. Mirlin, Phys. Rev. Lett. 98, 256801 (2007).

${ }^{13}$ S. Ryu, C. Mudry, H. Obuse, and A. Furusaki, Phys. Rev. Lett. 99, 116601 (2007).

${ }^{14}$ J. H. Bardarson, J. Tworzydlo, P. W. Brouwer, and C. W. J. Beenakker, Phys. Rev. Lett. 99, 106801 (2007).

${ }^{15}$ K. Nomura, M. Koshino, and S. Ryu, Phys. Rev. Lett. 99, 146806 (2007).

${ }^{16}$ See P. M. Ostrovsky, I. V. Gornyi, and A. D. Mirlin, Phys. Rev. B 74, 235443 (2006), and references therein.

${ }^{17}$ V. M. Edelstein, Zh. Eksp. Teor. Fiz. 95, 2151 (1989) [Sov. Phys. JETP 68, 1244 (1989)].

${ }^{18}$ V. M. Edelstein, Phys. Rev. Lett. 75, 2004 (1995).

${ }^{19}$ L. P. Gor'kov and E. I. Rashba, Phys. Rev. Lett. 87, 037004 (2001).

${ }^{20}$ S. K. Yip, Phys. Rev. B 65, 144508 (2002).
${ }^{21}$ P. A. Frigeri, D. F. Agterberg, A. Koga, and M. Sigrist, Phys. Rev. Lett. 92, 097001 (2004).

${ }^{22}$ M. Sigrist, D. F. Agterberg, P. A. Frigeri, N. Hayashi, R. P. Kaur, A. Koga, I. Milat, K. Wakabayashi, and Y. Yanase, J. Magn. Magn. Mater. 310, 536 (2007).

${ }^{23}$ K. V. Samokhin, E. S. Zijlstra, and S. K. Bose, Phys. Rev. B 69, 094514 (2004); 70, 069902(E) (2004).

${ }^{24}$ K. V. Samokhin, Phys. Rev. B 70, 104521 (2004).

${ }^{25}$ R. P. Kaur, D. F. Agterberg, and M. Sigrist, Phys. Rev. Lett. 94, 137002 (2005).

${ }^{26}$ C. L. Kane and E. J. Mele, Phys. Rev. Lett. 95, 226801 (2005).

${ }^{27}$ C. L. Kane and E. J. Mele, Phys. Rev. Lett. 95, 146802 (2005).

${ }^{28}$ B. A. Bernevig and S.-C. Zhang, Phys. Rev. Lett. 96, 106802 (2006).

${ }^{29}$ B. A. Bernevig, T. L. Hughes, and S.-C. Zhang, Science 314, 1757 (2006).

${ }^{30}$ M. König, S. Wiedmann, C. Brüne, A. Roth, H. Buhmann, L. W. Molenkamp, X.-L. Qi, and S.-C. Zhang, Science 318, 766 (2007).

${ }^{31}$ A. P. Schnyder, S. Ryu, A. Furusaki, and A. W. W. Ludwig, Phys. Rev. B 78, 195125 (2008).

${ }^{32}$ X.-L. Qi, T. L. Hughes, S. Raghu, and S.-C. Zhang, Phys. Rev. Lett. 102, 187001 (2009).

${ }^{33}$ A. Kitaev, arXiv:0901.2686, in Proceedings of the L.D. Landau Memorial Conference "Advances in Theoretical Physics," Chernogolovka, Moscow region, Russia, 22-26 June 2008 (unpublished).

${ }^{34} \mathrm{R}$. Roy, arXiv:cond-mat/0608064 (unpublished); arXiv:0803.2868 (unpublished).

${ }^{35}$ X. Qi, T. Hughes, and S. Zhang, Phys. Rev. B 81, 134508 (2010).

${ }^{36}$ S. Yip, arXiv:0910.0696 (unpublished).

${ }^{37}$ C. Caroli, P. G. de Gennes, and J. Matricon, Phys. Lett. 9, 307 (1964).

${ }^{38}$ R. Jackiw and P. Rossi, Nucl. Phys. B 190, 681 (1981).

${ }^{39}$ E. J. Weinberg, Phys. Rev. D 24, 2669 (1981).

${ }^{40}$ N. B. Kopnin and M. M. Salomaa, Phys. Rev. B 44, 9667 (1991).

${ }^{41}$ G. E. Volovik, JETP Lett. 57, 244 (1993); 70, 609 (1999). 
${ }^{42}$ N. Read and D. Green, Phys. Rev. B 61, 10267 (2000); N. Read, ibid. 79, 245304 (2009).

${ }^{43}$ D. A. Ivanov, Phys. Rev. Lett. 86, 268 (2001).

${ }^{44}$ C.-K. Lu and S. Yip, Phys. Rev. B 78, 132502 (2008).

${ }^{45}$ M. Sato and S. Fujimoto, Phys. Rev. B 79, 094504 (2009).

${ }^{46}$ Yukio Tanaka, Takehito Yokoyama, Alexander V. Balatsky, and Naoto Nagaosa, Phys. Rev. B 79, 060505(R) (2009).

${ }^{47}$ L. Fu and C. L. Kane, Phys. Rev. Lett. 100, 096407 (2008).

${ }^{48}$ P. Lee, arXiv:0907.2681 (unpublished).

${ }^{49}$ J. Sau, R. Lutchyn, S. Tewari, and S. Das Sarma, Phys. Rev. Lett. 104, 040502 (2010).

${ }^{50}$ Changing our convention $\alpha>0$ to $\alpha<0$ amounts to exchanging the helicities through $g_{k} \rightarrow-g_{k}$.

${ }^{51}$ M. I. Dyakonov and V. I. Perel, JETP Lett. 13, 467 (1971); Phys. Lett. A 35, 459 (1971).

${ }^{52}$ J. E. Hirsch, Phys. Rev. Lett. 83, 1834 (1999).

${ }^{53}$ S. Raghu, S. Chung, X. Qi, and S. Zhang, Phys. Rev. Lett. 104, 116401 (2010).

${ }^{54}$ T. Takimoto and P. Thalmeier, J. Phys.: Conf. Ser. 150, 042202
(2009).

${ }^{55}$ K. V. Samokhin and V. P. Mineev, Phys. Rev. B 77, 104520 (2008).

${ }^{56}$ W. Kohn and J. M. Luttinger, Phys. Rev. Lett. 15, 524 (1965).

${ }^{57}$ L. S. Braginskii and M. V. Entin, JETP Lett. 51, 660 (1990).

${ }^{58}$ N. Reyren, S. Thiel, A. D. Caviglia, L. Fitting Kourkoutis, G. Hammerl, C. Richter, C. W. Schneider, T. Kopp, A.-S. Rüetschi, D. Jaccard, M. Gabay, D. A. Muller, J.-M. Triscone, and J. Mannhart, Science 317, 1196 (2007).

${ }^{59}$ K. Ueno, S. Nakamura, H. Shimotani, A. Ohtomo, N. Kimura, T. Nojima, H. Aoki, Y. Iwasa, and M. Kawasaki, Nature Mater. 7, 855 (2008).

${ }^{60}$ B. Béri, arXiv:0909.5680 (unpublished).

${ }^{61}$ I. M. Khaymovich, N. B. Kopnin, A. S. Mel'nikov, and I. A. Shereshevskii, Phys. Rev. B 79, 224506 (2009).

${ }^{62}$ Roman Jackiw and So-Young Pi have solved the BdG Hamiltonian (6.20a) for a generic profile of the vortex $\Delta_{t}(r)$ in the $r$ representation. They also find a single normalizable Majorana state iff $\mu>0$ (private communication). 